\title{
Anorogenic alkaline granites from northeastern Brazil: major, trace, and rare earth elements in magmatic and metamorphic biotite and Na-mafic minerals ${ }^{\text {is }}$
}

\author{
J. Plá Cid ${ }^{\mathrm{a}, *}$, L.V.S. Nardi ${ }^{\mathrm{a}}$, H. Conceição ${ }^{\mathrm{b}}$, B. Bonin ${ }^{\mathrm{c}}$ \\ ${ }^{a}$ Curso de Pós-Graduação em in Geociências UFRGS. Campus da Agronomia-Inst. de Geoc., Av. Bento Gonçalves, 9500, 91509-900 CEP RS Brazil \\ ${ }^{\mathrm{b}}$ CPGG-PPPG/UFBA. Rua Caetano Moura, 123, Instituto de Geociências-UFBA, CEP-40210-350, Salvador-BA Brazil \\ ${ }^{c}$ Departement des Sciences de la Terre, Laboratoire de Pétrographie et Volcanologie-Université Paris-Sud. Centre d'Orsay, Bat. 504, F-91504, Paris, France
}

Accepted 29 August 2000

\begin{abstract}
The anorogenic, alkaline silica-oversaturated Serra do Meio suite is located within the Riacho do Pontal fold belt, northeast Brazil. This suite, assumed to be Paleoproterozoic in age, encompasses metaluminous and peralkaline granites which have been deformed during the Neoproterozoic collisional event. Preserved late-magmatic to subsolidus amphiboles belong to the riebeckite-arfvedsonite and riebeckitewinchite solid solutions. Riebeckite-winchite is frequently rimmed by $\mathrm{Ti}$-aegirine. Ti-aegirine cores are strongly enriched in $\mathrm{Nb}, \mathrm{Y}, \mathrm{Hf}$, and REE, which significantly decrease in concentrations towards the rims. REE patterns of Ti-aegirine are strikingly similar to Ti-pyroxenes from the Ilímaussaq peralkaline intrusion. Recrystallisation of mineral assemblages was associated with deformation although some original grains are still preserved. Magmatic annite was converted into magnetite and biotite with lower Fe/(Fe $+\mathrm{Mg})$ ratios. Recrystallised amphibole is pure riebeckite. Magmatic Ti-Na-bearing pyroxene was converted to low-Ti aegirine + titanite \pm astrophyllite/aenigmatite. The reaction riebeckite + quartz $\rightarrow$ aegirine + magnetite + quartz + fluid is also observed. Biotite and Na-mafic minerals recrystallised under metamorphic oxidising conditions corresponding to temperatures of $600^{\circ} \mathrm{C}$ between the NiNiO and $\mathrm{HM}$ buffers. C) 2001 Elsevier Science Ltd. All rights reserved.
\end{abstract}

Keywords: Anorogenic alkaline granites; Earth elements; Northeastern Brazil

\section{Introduction}

Granites related to the alkaline series are common in within-plate, anorogenic (Murthy and Venkatenaman, 1964; Martin and Piwinskii, 1972), or post orogenic settings (Nardi and Bonin, 1991). Classical alkaline anorogenic suites are exemplified by the Younger Granite province of Niger - Nigeria (Jacobson et al., 1958), the Proterozoic Gardar province, South Greenland (Upton, 1974), and the Finnish rapakivi magmatism (Vorma, 1976). Examples of post-orogenic alkaline suites are the Permian-Triassic Western Mediterranean Province Bonin (1980), Neoproterozoic Saibro Intrusive Suite, south Brazil (Nardi and Bonin, 1991), and the Pan-African Arabian Shield. Comparing both post-orogenic and anoro-

\footnotetext{
This paper is part of the Special Issue: Alkaline and Carbonatitic Magmatism and Associated Mineralization-Part II. Guest Editors: L.G. Gwalani, J.L. Lytwyn.

* Corresponding author.

E-mail address: placid@ @if.ufrgs.br (J. Plá Cid).
}

genic alkaline suites, Rogers and Greenberg (1990) showed that post-orogenic suites are slightly richer in $\mathrm{CaO}$ and $\mathrm{MgO}$ with lower amounts of alkalis. Peralkaline types are more abundant in anorogenic suites while metaluminous types are largely dominant in post-orogenic associations (Nardi and Bonin, 1991).

In this paper, major, trace and rare earth elements data on mafic minerals from a Paleoproterozoic anorogenic alkaline suite are presented and discussed. Trace and rare earth element data in sodic amphibole and pyroxene were obtained by ion microprobe, through the SIMS technical approach, showing the variation of these elements between magmatic and metamorphic grains.

\section{Geological setting}

Paleoproterozoic alkaline magmatism with potassic affinities have been described in the São Francisco Craton by Conceição (1990), Conceição (1994), Rosa (1994), Rios (1997) and Paim (1998). This potassic alkaline character 
is ascribed to the peculiar composition of the Paleoproterozoic mantle in northeastern Brazil, which has produced alkaline magmas with compositions suggesting metasomatized mantle sources.

The Serra do Meio Suite (SMS, Leite, 1997) is located in the Riacho do Pontal Fold Belt (RPFB, Brito Neves, 1975), in the Borborema Province of northeastern region of Brazil (Fig. 1). The RPFB, located in the northwestern border of the São Francisco Craton (SFC), is one of the several Brasiliano (1.0-0.45 Ga, Wernick, 1981; Barbosa and Dominguez, 1996) fold belts surrounding this craton. According to Jardim de Sá (1994), the Neoproterozoic fold belts in northeastern Brazil were generated during intracontinental collisional events, with no evidence of coeval subduction.

The Serra do Meio Suite is an alkaline granitic magmatism, which is part of the Campo Alegre de Lourdes Alkaline Province (Conceição, 1990). These alkaline granites have been studied by Leite (1987, 1997), Conceição (1990), Plá Cid (1994) and Plá Cid et al., (2000). Isotopic determinations at Campo Alegre de Lourdes Alkaline Province point to magmatic ages related with the Transamazônico Event $(2.0 \pm 0.2 \mathrm{Ga}$, Wernick, 1981), as evidenced by $\mathrm{U}-\mathrm{Pb}$ data in zircon/baddeleyite grains of the Angico dos Dias Carbonatite $(2.01 \mathrm{Ga}$, Silva et al., 1988). In this area, the end of Transamazônico Event was marked by crustal extension in a continental rift setting (Leite et al., 1993; Plá Cid, 1994), emplacement of the Angico do Dias carbonatite complex, outpouring of tholeitic to transitional basalts with cumulative preserved structures (Couto, 1989) and associated alkaline granites.

The Neoproterozoic event at Campo Alegre de Lourdes region was characterised by frontal collision of continental blocks, with extensive thrust tectonics along ENE-WSW striking surfaces (Leite et al., 1993; Plá Cid, 1994 Fig. 1C). NS-striking sub-vertical transcurrent zones were identified in the northeastern part of this region (Fig. 1C), and interpreted as lateral ramps active during the frontal event (Leite, 1997). All geological units were affected by this tectonic event which produced ductile shear structures along thrust planes and lateral ramps. Such a framework follows Plá Cid (1994) in that lithological contacts in the Serra do Meio Suite result from strong superimposition of tectonic regimes during the Neoproterozoic event and do not represent the igneous geometry.

\section{Geology and petrography}

The oldest rocks are represented by the late Archean Gneissic-Migmatitic Complex which yields a whole-rock $\mathrm{Rb}-\mathrm{Sr}$ age of $2.6 \mathrm{Ga}$ as determined by whole-rock $\mathrm{Rb}-\mathrm{Sr}$ isotopic dating (Dalton de Souza et al., 1979). These gneisses have granite and trondhjemite-tonalite compositions with development of migmatitic structures and were metamorphosed within the amphibolite facies. Metasedimentary rocks are represented by quartz-mica schist and calcareous schist belonging to Paleoproterozoic Serra da Boa Esperança Unit (Silva et al., 1988) and by metapelitic
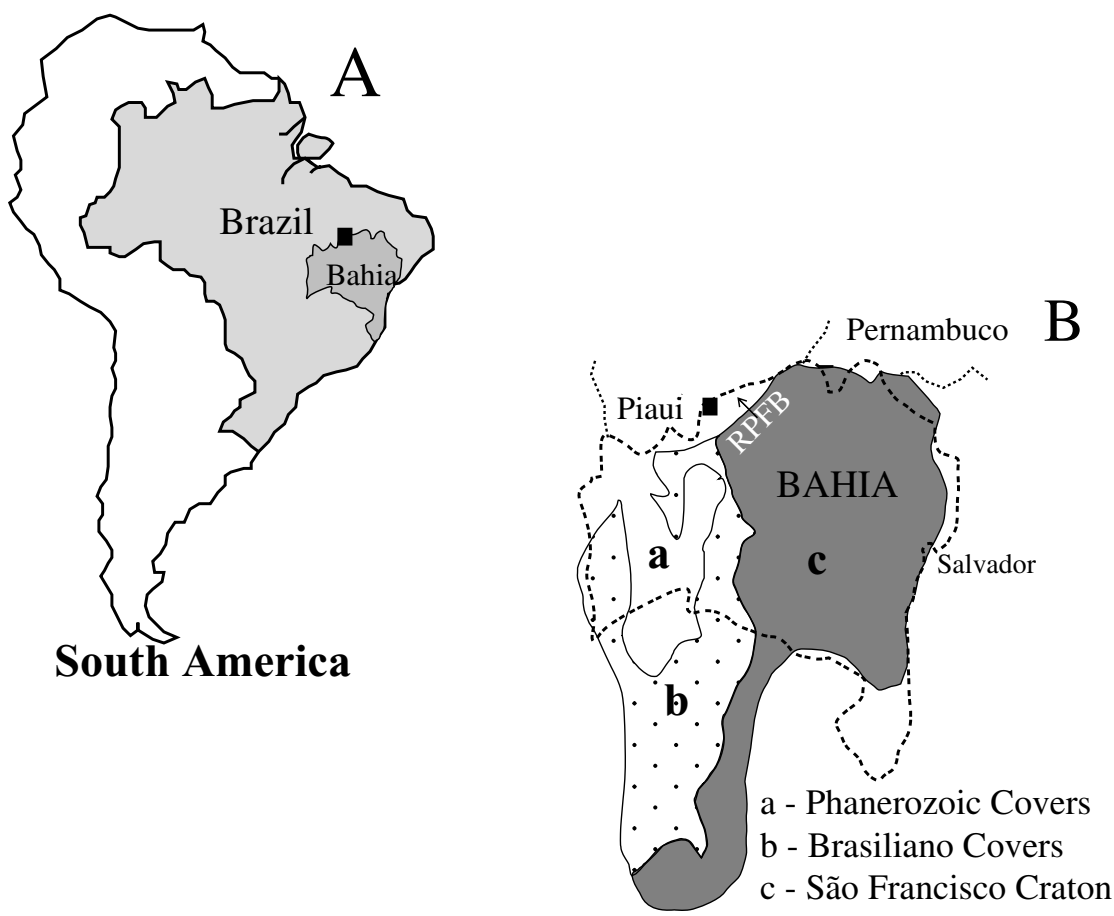

Fig. 1. (A) Location of studied area in South America. (B) The São Francisco Craton is within the state of Bahia. The Serra do Meio suite is located between Bahia and Piauí states, inside the RPFB terrain. (C) Geological map of the Serra do Meio suite, modified after Leite (1997). 


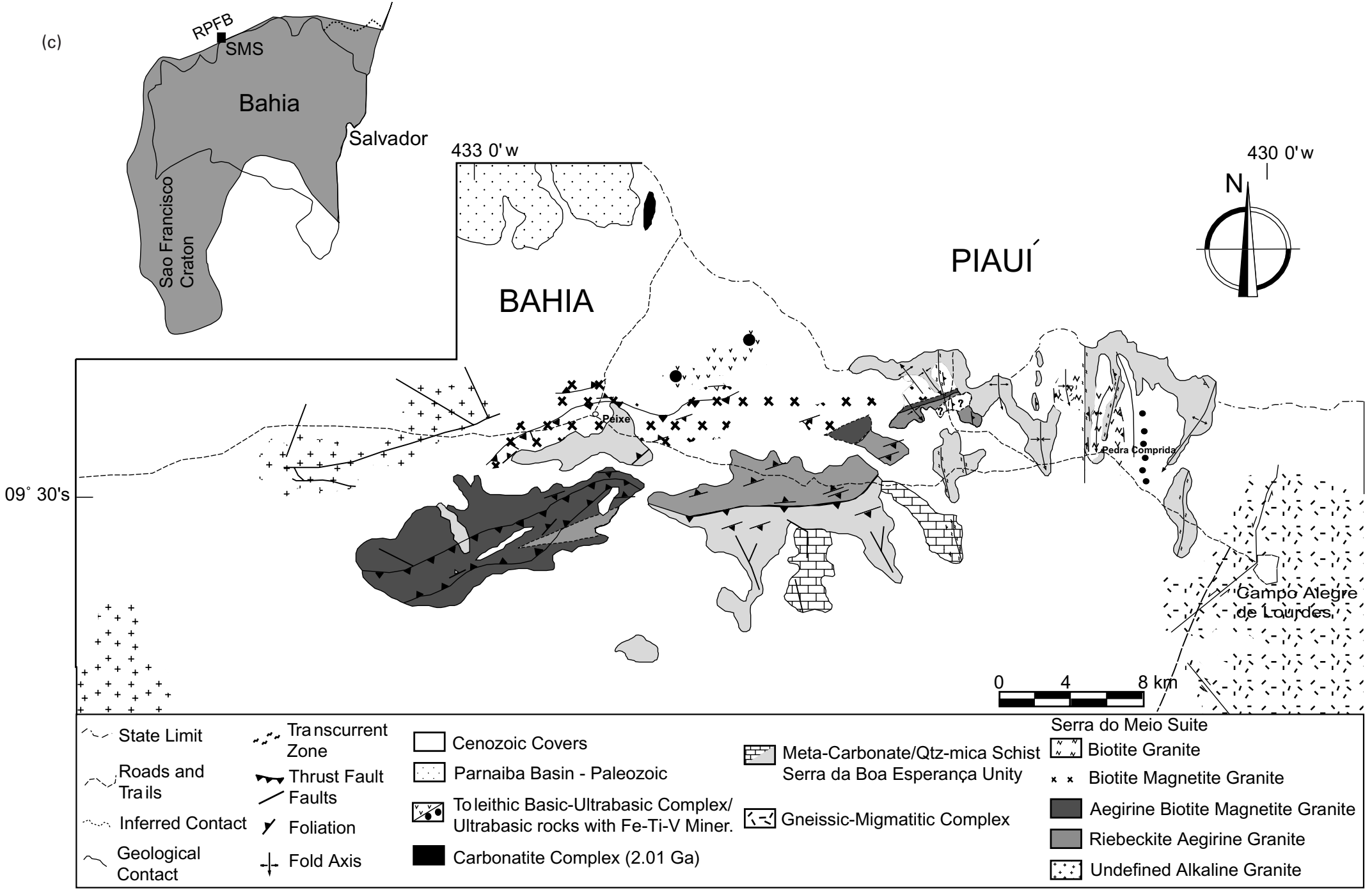

Fig. 1. (continued) 


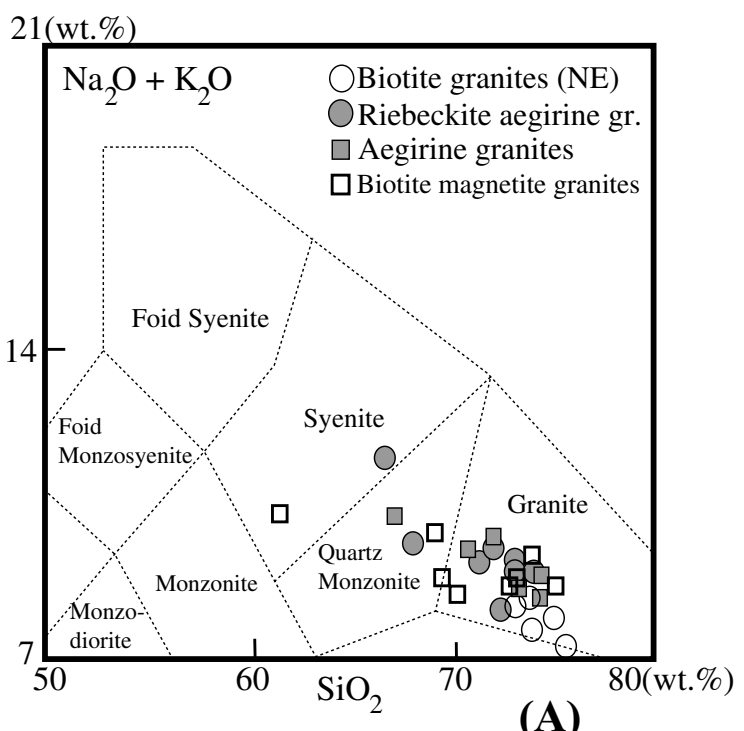

(A)
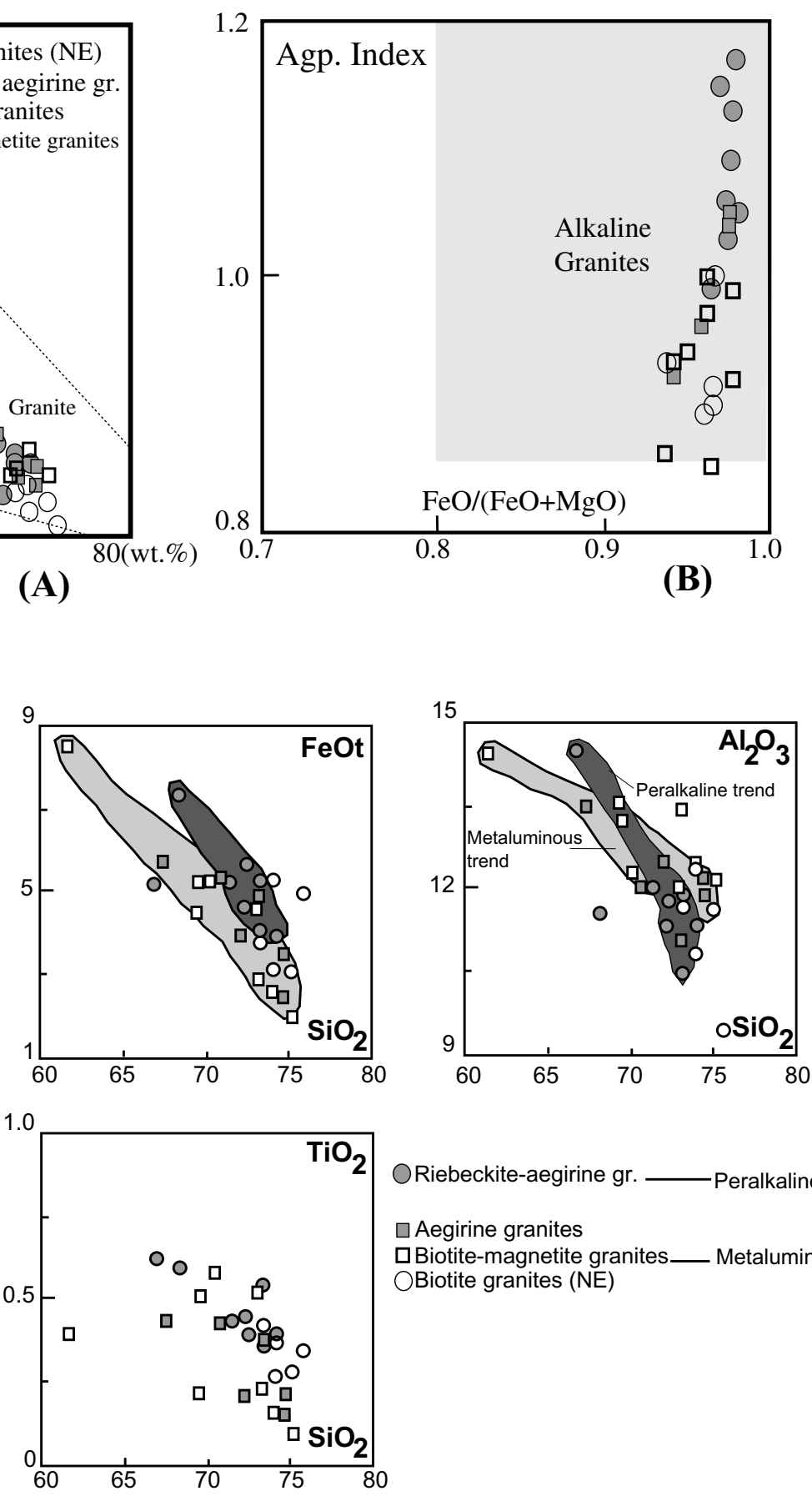

(C)

Fig. 2. (A) Total alkalis vs. silica (TAS) diagram, in wt.\%, after Le Maitre et al. (1989), with chemical classification and nomenclature of plutonic rocks, according to Middlemost (1994). (B) Agpaitic index vs. FeOt/(FeOt $+\mathrm{MgO}$ ) diagram (Nardi, 1991), showing the usual field for alkaline granites. (C) Harker diagrams of the Serra do Meio Suite.

and metapsamitic rocks included in the Mesoproterozoic Santo Onofre Group (Leite, 1997), both metamorphosed to greenschist facies.

The Serra do Meio Suite includes several intrusions oriented along ENE-WSW trends (Fig. 1C) that intrude the Serra da Boa Esperança Unit. Quartz-mica schist xenoliths are abundant within the alkaline granites. The granites were deformed by the Brasiliano collision that produced gneissic structures with a marked foliation dipping to the SE or NW (Plá Cid et al., 2000). The preBrasiliano age of alkaline granites is suggested by wholerock $\mathrm{Rb}-\mathrm{Sr}$ errorchrons yielding an age of ca. $850 \mathrm{Ma}$ 
Table 1

Representative analyses from Serra do Meio Suite

\begin{tabular}{|c|c|c|c|c|c|c|c|c|c|c|}
\hline $\begin{array}{l}\text { Sample } \\
\text { Facies }\end{array}$ & $\begin{array}{l}\text { CL-05b } \\
\text { NE Met. }\end{array}$ & $\begin{array}{l}\text { CL-54 } \\
\text { NE Met. }\end{array}$ & $\begin{array}{l}\text { CL-55 } \\
\text { NE Met. }\end{array}$ & $\begin{array}{l}\text { CL-87 } \\
\text { Slight Peralk. }\end{array}$ & $\begin{array}{l}\text { GA-68 } \\
\text { Slight Peralk. }\end{array}$ & $\begin{array}{l}\text { JP-49 } \\
\text { Slight Peralk. }\end{array}$ & $\begin{array}{l}\text { GA-34 } \\
\text { Strong Peralk. }\end{array}$ & $\begin{array}{l}\text { GA-46 } \\
\text { Strong Peralk. }\end{array}$ & $\begin{array}{l}\text { GA-46a } \\
\text { Strong Peralk. }\end{array}$ & $\begin{array}{l}\text { PPB-78a } \\
\text { Strong Peralk. }\end{array}$ \\
\hline $\mathrm{SiO}_{2}$ & 75.10 & 75.70 & 73.30 & 73.20 & 74.50 & 67.30 & 74.20 & 71.40 & 72.30 & 73.30 \\
\hline $\mathrm{TiO}_{2}$ & 0.28 & 0.34 & 0.42 & 0.37 & 0.15 & 0.44 & 0.39 & 0.43 & 0.44 & 0.36 \\
\hline $\mathrm{Al}_{2} \mathrm{O}_{3}$ & 11.60 & 9.40 & 11.60 & 11 & 12.10 & 13.40 & 11.30 & 12 & 11.30 & 11.80 \\
\hline $\mathrm{Fe}_{2} \mathrm{O}_{3}$ & 1 & 3 & 1.30 & 3.70 & 0.64 & 4.30 & 2.70 & 3.70 & 3 & 3.20 \\
\hline $\mathrm{FeO}$ & 2.10 & 2.20 & 2.50 & 1.50 & 1.80 & 1.80 & 1.40 & 1.80 & 1.90 & 1.10 \\
\hline $\mathrm{MgO}$ & 0.10 & 0.16 & 0.23 & 0.12 & 0.10 & 0.14 & 0.10 & 0.10 & 0.10 & 0.10 \\
\hline $\mathrm{CaO}$ & 0.89 & 0.46 & 0.89 & 0.45 & 0.79 & 1.30 & 0.40 & 0.64 & 0.58 & 0.31 \\
\hline $\mathrm{Na}_{2} \mathrm{O}$ & 3.70 & 3.10 & 3.50 & 3.90 & 3.80 & 5.10 & 4.30 & 4.80 & 4.60 & 4.10 \\
\hline $\mathrm{K}_{2} \mathrm{O}$ & 4.10 & 4 & 4.60 & 4.60 & 5 & 5.10 & 4.50 & 4.30 & 4.80 & 5 \\
\hline $\mathrm{MnO}$ & 0.13 & 0.17 & 0.11 & 0.21 & 0.06 & 0.16 & 0.21 & 0.18 & 0.22 & 0.13 \\
\hline $\mathrm{P}_{2} \mathrm{O}_{5}$ & 0.05 & 0.05 & 0.05 & 0.05 & 0.05 & 0.05 & 0.05 & 0.05 & 0.05 & 0.05 \\
\hline $\mathrm{H}_{2} \mathrm{Op}$ & 0.30 & 0.54 & 0.30 & 0.23 & 0.35 & 0.11 & 0.38 & 0.26 & 0.19 & 0.55 \\
\hline $\mathrm{CO}_{2}$ & 0.53 & 0.37 & 0.93 & 0.43 & 0.48 & 0.64 & 0.05 & 0.19 & 0.45 & 0.05 \\
\hline Total & 99.88 & 99.49 & 99.73 & 99.76 & 99.82 & 99.84 & 99.98 & 99.85 & 99.93 & 100.05 \\
\hline $\mathrm{F}$ & 1700 & 1100 & 1200 & 930 & 1800 & 2300 & 820 & 1300 & 810 & 250 \\
\hline $\mathrm{Cl}$ & 20 & 20 & 20 & 20 & 20 & 20 & 20 & 20 & 20 & 20 \\
\hline Ag. Ind. & 0.91 & 1 & 0.93 & 1.04 & 0.96 & 1.04 & 1.06 & 1.05 & 1.13 & 1.03 \\
\hline $\mathrm{FeOt}$ & 3 & 4.90 & 3.67 & 4.83 & 2.38 & 5.67 & 3.83 & 5.13 & 4.60 & 3.98 \\
\hline $\mathrm{Ba}$ & 840 & 200 & 390 & 240 & 160 & 710 & 440 & 510 & 340 & 300 \\
\hline $\mathrm{Nb}$ & 81 & 350 & 130 & 200 & 120 & 190 & 53 & 77 & 50 & 56 \\
\hline $\mathrm{Cs}$ & 6 & 5 & 5 & 5 & 5 & 5 & 6 & 5 & 5 & 5 \\
\hline $\mathrm{Rb}$ & 110 & 160 & 82 & 130 & 190 & 150 & 68 & 88 & 72 & 84 \\
\hline $\mathrm{Hf}$ & 15 & 68 & 27 & 34 & 10 & 21 & 9 & 12 & 8 & 8 \\
\hline $\mathrm{Sr}$ & 68 & 29 & 73 & 36 & 35 & 140 & 33 & 40 & 19 & 13 \\
\hline $\mathrm{Y}$ & 120 & 270 & 95 & 160 & 170 & 110 & 43 & 80 & 44 & 37 \\
\hline $\mathrm{Zr}$ & 720 & 2690 & 1060 & 1440 & 430 & 810 & 360 & 600 & 340 & 400 \\
\hline $\mathrm{Ga}$ & 32 & 29 & 35 & 33 & 46 & 41 & 42 & 37 & 40 & 41 \\
\hline V & 8 & 8 & 8 & 8 & 5 & 8 & 8 & 8 & 8 & 8 \\
\hline Th & 16 & 45 & 18 & 27 & 27 & 22 & 5 & 6 & 5 & 5 \\
\hline $\mathrm{U}$ & 10 & 10 & 10 & 10 & 10 & 10 & 10 & 10 & 10 & 10 \\
\hline $\mathrm{Ta}$ & 7 & 26 & 5 & 11 & 10 & 13 & 5 & 5 & 5 & 5 \\
\hline $\mathrm{Cu}$ & 11 & 15 & 11 & 19 & 22 & 15 & 7 & 11 & 7 & 15 \\
\hline Co & 28 & 28 & 28 & 51 & 28 & 51 & 23 & 28 & 28 & 28 \\
\hline $\mathrm{Ni}$ & 29 & 18 & 41 & 18 & 59 & 29 & 129 & 47 & 18 & 24 \\
\hline $\mathrm{Cr}$ & 52 & 77 & 103 & 52 & 116 & 52 & 258 & 65 & 52 & 39 \\
\hline $\mathrm{La}$ & & & & 208.2 & & 155.1 & & 76.29 & & \\
\hline $\mathrm{Ce}$ & & & & 436.6 & & 335.2 & & 170.9 & & \\
\hline $\mathrm{Nd}$ & & & & 179.1 & & 144.4 & & 70.1 & & \\
\hline $\mathrm{Sm}$ & & & & 29.26 & & 24.32 & & 14.54 & & \\
\hline $\mathrm{Eu}$ & & & & 3.22 & & 2.61 & & 2.25 & & \\
\hline $\mathrm{Gd}$ & & & & 20.39 & & 18.26 & & 10.81 & & \\
\hline Dy & & & & 16.89 & & 16.23 & & 9.16 & & \\
\hline Ho & & & & 3.18 & & 3.18 & & 1.72 & & \\
\hline $\mathrm{Er}$ & & & & 7.6 & & 8.2 & & 4.08 & & \\
\hline $\mathrm{Yb}$ & & & & 5.15 & & 7.04 & & 3.3 & & \\
\hline $\mathrm{Lu}$ & & & & 0.62 & & 0.87 & & 0.44 & & \\
\hline REE & & & & 910.21 & & 715.41 & & 363.59 & & \\
\hline
\end{tabular}

which indicates that these granites have experienced resetting of the isotopic system.

Preserved igneous structures such as pod-like portions with isotropic coarse-grained texture surrounded by deformed portions, as well as biotite schlieren, are present within these granites in spite of Brasiliano deformation and metamorphism.

Modal analyses (Streckeisen, 1976) indicate that the Serra do Meio Suite is composed of alkali feldspar granites, and subordinate amounts of quartz alkali feldspar syenites. Normally these granites exhibit strong mineral orientation and recrystallisation textures but alkali feldspar phenocrysts and some mafic minerals are still preserved. Vein-type mesoperthitic alkali feldspar is the major magmatic felsic phase whereas quartz and the subsolvus assemblage (albite + microcline) constitute the granoblastic groundmass. Three different lithotypes were identified on petrographic grounds: $(\boldsymbol{i})$ metaluminous granites, sometimes 

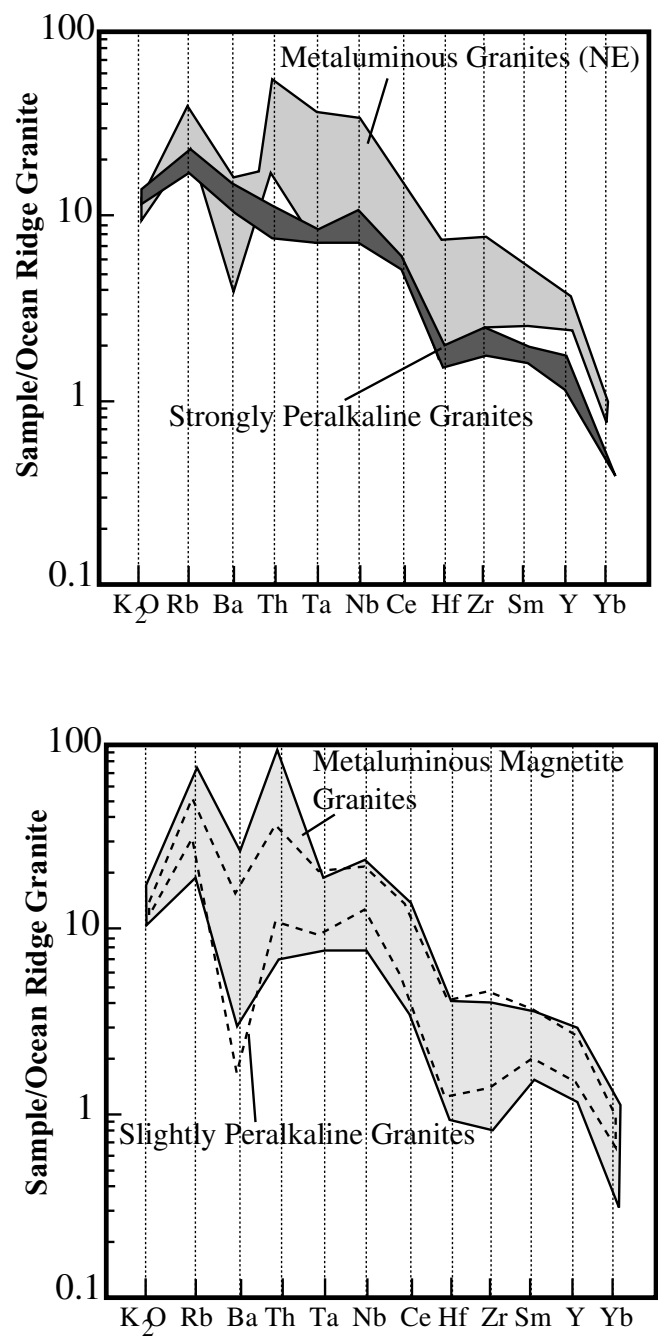

Fig. 3. Spidergrams of the Serra do Meio suite normalised to Ocean Ridge Granites (Pearce et al., 1984).

with magnetite porphyroblasts; (ii) slightly peralkaline granites, and (iii) strongly peralkaline granites.

The northeastern plutons, as well as restricted parts of the ENE-WSW striking bodies, are made up of metaluminous granites (Fig. 1). Biotite is the only mafic mineral, and occurs as interstitial grains or in millimetric elongated concentrations. Fluorite grains are common. In the ENEWSW striking bodies, biotite is sometimes transformed to muscovite and magnetite porphyroblasts are associated with interstitial carbonate which suggests the important role of $\mathrm{Fe}-\mathrm{CO}_{2}$-bearing fluids during the Brasiliano deformation (Plá Cid, 1994).

The slightly peralkaline granites have aegirine, aegirineaugite, and colourless pyroxene optically identified as hedenbergite (Plá Cid et al., 2000). Interstitial biotite and magnetite porphyroblasts are rare. The size, shape, and composition of hedenbergite and of aegirine-augite grains indicate their magmatic origin. Aegirine-augite constitutes either millimetre-size irregular concentrations or aciculate grains included in alkali feldspar cores (Conceição, 1990).
The recrystallised clear rims of alkali feldspar are devoid of pyroxene inclusions. Aegirine occurs as magmatic and metamorphic crystals without compositional differences. Poikiloblastic aegirine encloses alkali feldspar, albite, and quartz.

The strong peralkaline granites have amphibole, aegirine and aegirine-augite, aenigmatite or astrophyllite, biotite, titanite, and magnetite as mafic magmatic and metamorphic constituents:

\subsection{Metamorphic Minerals}

Amphibole occurs as dark- to brownish-blue poikiloblasts, and as interstitial grains. The interstitial grains are surrounded by aegirine along the foliation, and both were produced by recrystallisation during the Neoproterozoic event, whereas the poikiloblasts probably recrystallised later in the same metamorphic event (Leite et al., 1991). These poikiloblasts have a reddish fibrous mineral (aenigmatite or astrophyllite) and euhedral titanite along their borders (Plá Cid et al., 2000). The presence of magnetite blasts along the foliation reflects the occurrence of $\mathrm{Fe}$ bearing fluids associated with this metamorphism.

\subsection{Magmatic Minerals}

They are non oriented, subhedral, dark-amphibole grains sometimes mantled by aegirine and aegirine-augite. Subhedral pyroxene phenocrysts are intensively zoned and crosscut by the foliation (Plá Cid et al., 2000). Some subhedral, interstitial grains of pyroxene were also interpreted as magmatic.

\section{Geochemistry}

The alkaline affinity of the Serra do Meio Suite is shown in Fig. 2A and B. Additionally, the very low contents of $\mathrm{CaO}$ and $\mathrm{MgO}$, high concentrations of alkalis and the very high values of $\mathrm{FeOt} /(\mathrm{FeOt}+\mathrm{MgO})$ ratios and the agpaitic index are diagnostic of their alkaline affinity (Table 1; Sorensen, 1974; Bonin, 1982; Whalen et al., 1987; Rogers and Greenberg, 1990; Nardi, 1991, and references therein). Typical anorogenic suites and the Serra do Meio granites have similar compositions (Fig. 2B).

The agpaitic index, which varies between 0.85 and 1.0 in the metaluminous granites, reaches 1.05 in the slightly peralkaline granites and 1.17 in the strongly peralkaline types. As pointed out by Plá Cid et al. (2000), the Harker diagrams (Fig. 2C) illustrate that the Serra do Meio Suite evolved along two evolutionary paths represented by: (i) the metaluminous trend, formed by metaluminous and slightly peralkaline granites, and (ii) the peralkaline trend, composed by the strongly peralkaline rocks. The evolutionary trends can be generated by two alkaline parental liquids due to differences in their sources or petrogenetic processes. The peralkaline trend is richer in $\mathrm{Na}_{2} \mathrm{O}, \mathrm{TiO}_{2}$, and $\mathrm{FeOt}$, 

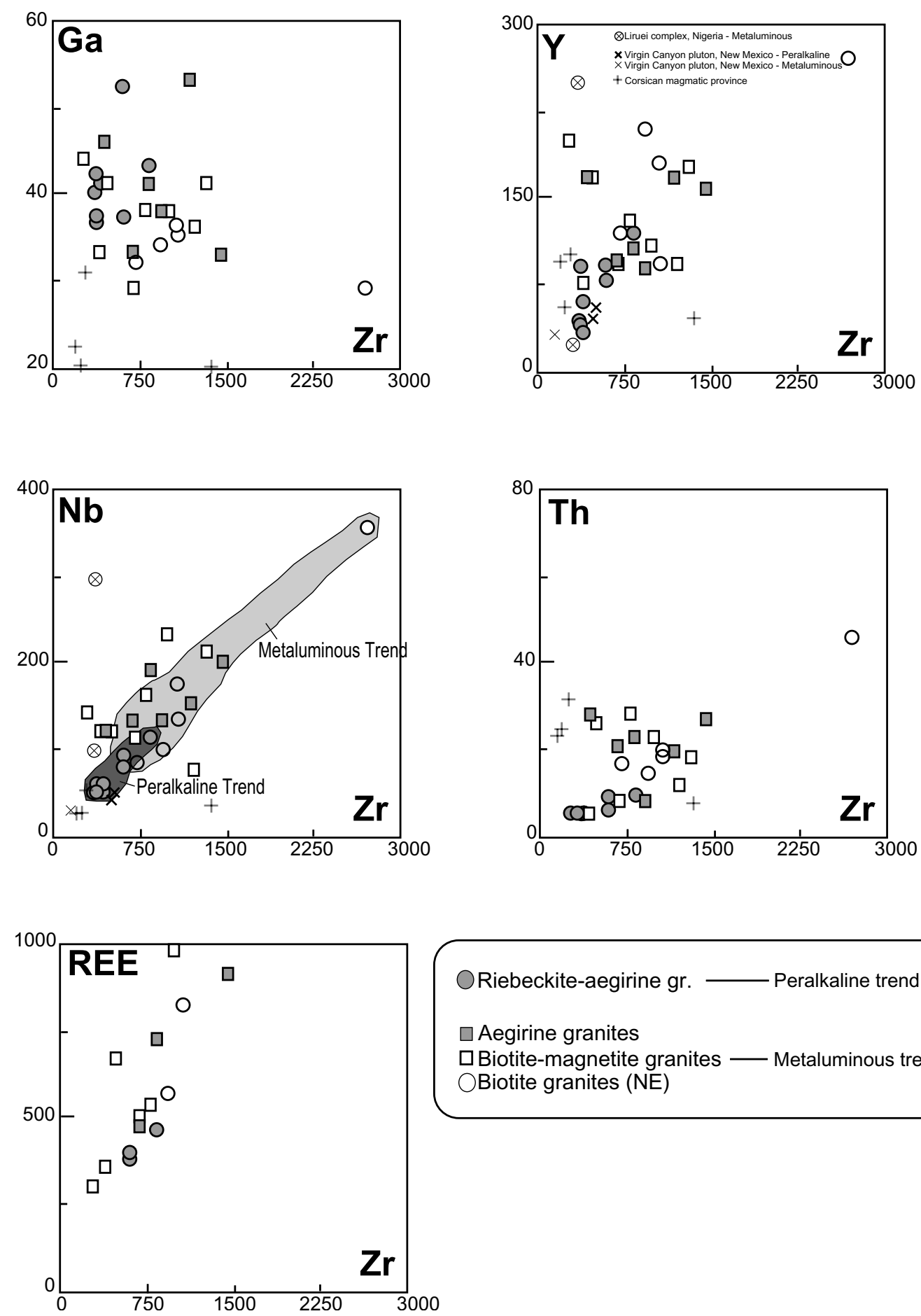

Riebeckite-aegirine gr. — Peralkaline trend

$\square$ Aegirine granites

$\square$ Biotite-magnetite granites — Metaluminous trend OBiotite granites (NE)

Fig. 4. Ga, Y, Nb, Th, and REE vs. Zr plots, showing the different concentrations for the peralkaline and metaluminous trends.

whereas the metaluminous one has higher concentration of $\mathrm{CaO}$ and $\mathrm{Al}_{2} \mathrm{O}_{3}$ (Fig. 2C). Their parallelism suggest a similar magmatic evolution controlled by feldspar fractionation as indicated by the $\mathrm{Al}_{2} \mathrm{O}_{3} \times \mathrm{SiO}_{2}$ plot.

The whole-rock trace element concentration of the Serra do Meio Suite are comparable to those of typical anorogenic granites (Fig. 3). They are characterised by high concentration of incompatible HFS elements (Nb, Zr, Ga, Y, Hf), light rare earth elements (LREE; Table 1), and $\mathrm{Rb} / \mathrm{Sr}$ ratios $>1$. As previously observed by Plá Cid et al. (1997), Zr exhibits a peculiar behaviour, with higher concentration in granites of the metaluminous trend than in the more peralkaline varieties (Fig. 4). The $\mathrm{Zr}$ solubility increases with the peralkalinity (Watson, 1979) and alkali contents of the magma (Harris, 1980). $\mathrm{Zr}$ enrichment in metaluminous granites, followed by higher concentration of HFSE and 

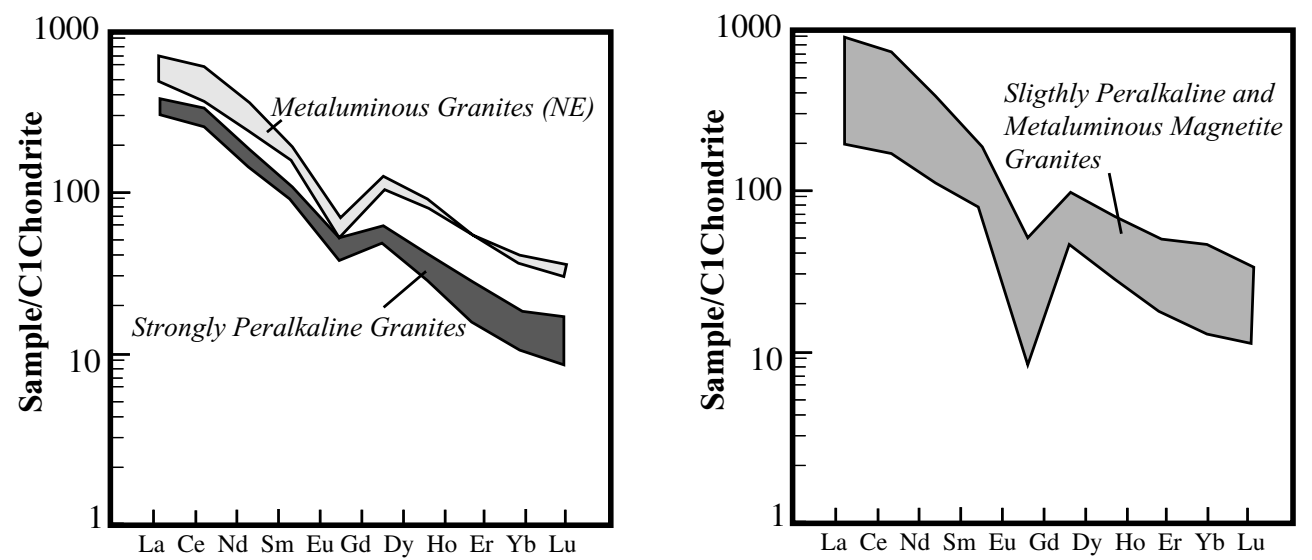

Fig. 5. REE patterns of the Serra do Meio suite normalised to the chondritic values (C1) of Evensen et al. (1978).

REE (Fig. 4), are opposite of the expected trend in alkaline magmas. According to Plá Cid et al. (2000), this can be explained by the higher F-contents observed in the metaluminous liquid which probably promotes the stabilisation of F-HFSE complexes (Harris, 1980). An HFSE and REEenriched source is therefore assumed for metaluminous magmas.

The REE patterns of metaluminous and peralkaline granites have similar shapes (Fig. 5). They are depleted in HREE relative to LREE, suggesting residual garnet in the source, and exhibit Eu-negative anomalies. The peralkaline trend exhibit slightly more negative Eu-anomalies than those of the metaluminous trend. The different behaviour of $\mathrm{Eu}, \mathrm{Rb}$, $\mathrm{Sr}$ and $\mathrm{Ba}$ in the metaluminous trend, relative to the strongly peralkaline granites, was ascribed to more intense alkali feldspar fractionation during magmatic evolution in the metaluminous trend (Plá Cid et al., 2000).

\section{Mineralogy}

Previous mineralogical studies in the Serra do Meio Suite

Table 2

Representative analysis of micas from Serra do Meio suite. Crystals in contact with magnetite porphyroblasts (rim-mt)

\begin{tabular}{|c|c|c|c|c|c|c|c|c|c|c|c|c|}
\hline \multirow{2}{*}{$\begin{array}{l}\text { Facies } \\
\text { Location }\end{array}$} & \multicolumn{2}{|c|}{ Slightly peralkaline } & \multicolumn{2}{|c|}{ Biotite granite (NE) } & \multicolumn{2}{|c|}{ Biotite magnetite granite } & \multicolumn{2}{|c|}{ Strongly peralkaline } & \multicolumn{4}{|c|}{ Metamorphic grains } \\
\hline & Core & Rim & Rim & Core & Core & Core & Core & Rim & Core & Rim & Rim-mt & Rim-mt \\
\hline $\mathrm{SiO}_{2}$ & 35.66 & 35.45 & 34.14 & 33.14 & 35.28 & 35.35 & 34.53 & 35.09 & 36.69 & 36.53 & 36.19 & 36.32 \\
\hline $\mathrm{TiO}_{2}$ & 2.80 & 2.78 & 3.00 & 2.65 & 2.84 & 3.08 & 2.55 & 2.39 & 2.39 & 2.35 & 2.34 & 2.44 \\
\hline $\mathrm{Al}_{2} \mathrm{O}_{3}$ & 14.59 & 14.34 & 15.34 & 14.95 & 14.43 & 13.50 & 10.12 & 10.35 & 10.32 & 10.32 & 10.26 & 10.24 \\
\hline $\mathrm{FeO}$ & 31.09 & 31.20 & 32.26 & 31.81 & 31.02 & 31.58 & 33.78 & 34.04 & 28.46 & 28.55 & 26.67 & 26.78 \\
\hline $\mathrm{MnO}$ & 0.28 & 0.29 & 0.27 & 0.35 & 0.50 & 0.68 & 1.80 & 1.95 & 1.42 & 1.54 & 1.74 & 1.73 \\
\hline $\mathrm{MgO}$ & 2.03 & 2.20 & 0.88 & 0.89 & 2.02 & 1.96 & 2.03 & 1.91 & 5.44 & 5.60 & 6.64 & 6.54 \\
\hline $\mathrm{CaO}$ & 0.11 & 0.00 & 0.00 & 0.00 & 0.00 & 0.00 & 0.16 & 0.46 & 0.03 & 0.00 & 0.00 & 0.00 \\
\hline $\mathrm{Na}_{2} \mathrm{O}$ & 0.08 & 0.00 & 0.00 & 0.00 & 0.13 & 0.00 & 0.00 & 0.00 & 0.00 & 0.00 & 0.00 & 0.02 \\
\hline $\mathrm{K}_{2} \mathrm{O}$ & 8.99 & 9.19 & 9.33 & 9.46 & 9.50 & 9.18 & 8.10 & 7.02 & 9.11 & 9.29 & 9.50 & 9.22 \\
\hline F & 0.31 & 0.42 & 0.12 & 0.38 & 0.21 & 0.31 & 0.41 & 0.00 & 0.83 & 0.47 & 0.73 & 0.91 \\
\hline $\mathrm{H}_{2} \mathrm{O}$ & 1.63 & 1.57 & 1.70 & 1.53 & 1.67 & 1.61 & 1.49 & 1.69 & 1.36 & 1.53 & 1.40 & 1.32 \\
\hline Total & 97.57 & 97.44 & 97.04 & 95.16 & 97.60 & 97.25 & 94.96 & 94.90 & 96.06 & 96.18 & 95.47 & 95.52 \\
\hline O_F & 0.13 & 0.18 & 0.05 & 0.16 & 0.09 & 0.13 & 0.17 & 0 & 0.35 & 0.2 & 0.31 & 0.38 \\
\hline Ctotal & 97.44 & 97.26 & 96.99 & 95 & 97.51 & 97.12 & 94.79 & 94.9 & 95.71 & 95.98 & 95.16 & 95.14 \\
\hline $\mathrm{Si}$ & 5.777 & 5.776 & 5.606 & 5.602 & 5.735 & 5.793 & 5.931 & 5.950 & 6.083 & 6.027 & 6.011 & 6.039 \\
\hline AlIV & 2.223 & 2.224 & 2.394 & 2.398 & 2.265 & 2.207 & 2.047 & 2.050 & 1.917 & 1.973 & 1.989 & 1.961 \\
\hline AlVI & 0.561 & 0.528 & 0.573 & 0.578 & 0.497 & 0.398 & 0.000 & 0.017 & 0.097 & 0.032 & 0.019 & 0.044 \\
\hline $\mathrm{Ti}$ & 0.341 & 0.341 & 0.370 & 0.337 & 0.347 & 0.380 & 0.329 & 0.304 & 0.298 & 0.291 & 0.293 & 0.305 \\
\hline $\mathrm{Fe}^{2}$ & 4.212 & 4.251 & 4.430 & 4.497 & 4.217 & 4.328 & 4.853 & 4.826 & 3.946 & 3.939 & 3.705 & 3.723 \\
\hline $\mathrm{Mn}$ & 0.038 & 0.040 & 0.038 & 0.050 & 0.069 & 0.094 & 0.262 & 0.279 & 0.200 & 0.216 & 0.244 & 0.244 \\
\hline $\mathrm{Mg}$ & 0.490 & 0.534 & 0.216 & 0.224 & 0.490 & 0.479 & 0.519 & 0.482 & 1.346 & 1.377 & 1.645 & 1.621 \\
\hline $\mathrm{Ca}$ & 0.019 & 0.000 & 0.000 & 0.000 & 0.000 & 0.000 & 0.030 & 0.084 & 0.006 & 0.000 & 0.000 & 0.000 \\
\hline $\mathrm{Na}$ & 0.025 & 0.000 & 0.000 & 0.000 & 0.041 & 0.000 & 0.000 & 0.000 & 0.000 & 0.000 & 0.000 & 0.006 \\
\hline $\mathrm{K}$ & 1.858 & 1.910 & 1.954 & 2.039 & 1.970 & 1.919 & 1.775 & 1.519 & 1.927 & 1.955 & 2.014 & 1.956 \\
\hline Cations & 15.54 & 15.6 & 15.58 & 15.73 & 15.63 & 15.6 & 15.75 & 15.51 & 15.82 & 15.81 & 15.92 & 15.9 \\
\hline $\mathrm{Fe} / \mathrm{Fe}+\mathrm{Mg}$ & 0.90 & 0.89 & 0.95 & 0.95 & 0.90 & 0.90 & 0.90 & 0.91 & 0.75 & 0.74 & 0.69 & 0.70 \\
\hline
\end{tabular}


Table 3

Representative analysis of amphiboles from Serra do Meio suite. Roman numbers represent the crystals zoned observed in Fig. 12

\begin{tabular}{|c|c|c|c|c|c|c|c|c|c|c|c|c|}
\hline \multirow{2}{*}{$\begin{array}{l}\text { Type } \\
\text { Location }\end{array}$} & \multicolumn{6}{|c|}{ Riebeckite-winchite (PPB-78A) I, VII, VIII, IV } & \multicolumn{3}{|c|}{ Riebeckite-arfvedsonite } & \multicolumn{3}{|c|}{ Recrystallised crystals } \\
\hline & Core & Core & Core & Core & Rim & Core & Core & Core & Core & Core & Rim & Core \\
\hline $\mathrm{SiO}_{2}$ & 48.36 & 49.66 & 48.33 & 49.61 & 50.44 & 50.02 & 52.31 & 52.01 & 52.18 & 52.68 & 52.94 & 51.81 \\
\hline $\mathrm{TiO}_{2}$ & 1.69 & 0.20 & 0.27 & 0.39 & 0.31 & 0.35 & 0.21 & 0.20 & 0.20 & 0.13 & 0.19 & 0.07 \\
\hline $\mathrm{Al}_{2} \mathrm{O}_{3}$ & 2.14 & 1.51 & 1.74 & 1.99 & 1.86 & 1.90 & 1.34 & 1.37 & 1.34 & 0.47 & 0.32 & 0.17 \\
\hline $\mathrm{MgO}$ & 0.48 & 0.48 & 0.62 & 0.61 & 0.55 & 0.57 & 2.08 & 1.99 & 1.95 & 0.82 & 0.60 & 0.34 \\
\hline $\mathrm{CaO}$ & 1.01 & 0.74 & 0.79 & 0.94 & 0.85 & 0.91 & 0.82 & 0.77 & 0.85 & 0.04 & 0.03 & 0.11 \\
\hline $\mathrm{MnO}$ & 1.11 & 0.84 & 0.74 & 0.84 & 0.84 & 1.06 & 0.79 & 0.86 & 0.88 & 0.31 & 0.28 & 0.44 \\
\hline $\mathrm{FeOt}$ & 35.48 & 36.07 & 34.90 & 35.30 & 35.52 & 34.74 & 31.47 & 31.42 & 32.20 & 36.97 & 36.97 & 37.21 \\
\hline $\mathrm{Na}_{2} \mathrm{O}$ & 5.94 & 5.93 & 5.78 & 5.88 & 5.82 & 5.82 & 6.74 & 6.85 & 6.76 & 6.77 & 6.69 & 6.61 \\
\hline $\mathrm{K}_{2} \mathrm{O}$ & 1.01 & 0.69 & 0.72 & 0.93 & 0.82 & 0.80 & 0.81 & 0.85 & 0.86 & 0.13 & 0.11 & 0.20 \\
\hline $\mathrm{F}$ & 0.00 & 0.00 & 0.00 & 0.00 & 0.00 & 0.40 & 0.29 & 0.37 & 0.29 & 0.06 & 0.00 & 0.05 \\
\hline $\mathrm{Cl}$ & 0.01 & 0.00 & 0.00 & 0.03 & 0.00 & 0.03 & 0.00 & 0.00 & 0.00 & 0.00 & 0.00 & 0.00 \\
\hline Subtotal & 97.23 & 96.13 & 93.89 & 96.52 & 97.00 & 96.60 & 96.86 & 96.69 & 97.51 & 98.38 & 98.13 & 97.01 \\
\hline O_F_Cl & 0.00 & 0.00 & 0.00 & 0.01 & 0.00 & 0.18 & 0.12 & 0.16 & 0.12 & 0.03 & 0.00 & 0.02 \\
\hline $\mathrm{H}_{2} \mathrm{O}$ & 1.85 & 1.84 & 1.80 & 1.84 & 1.86 & 1.64 & 0.00 & 0.00 & 0.00 & 1.86 & 1.89 & 1.85 \\
\hline Total & 99.08 & 97.97 & 95.69 & 98.35 & 98.86 & 98.06 & 96.74 & 96.53 & 97.39 & 100.21 & 100.02 & 98.84 \\
\hline TSi & 7.53 & 7.76 & 7.73 & 7.73 & 7.80 & 7.81 & 8.08 & 8.07 & 8.03 & 7.99 & 8.05 & 8.01 \\
\hline Tal & 0.39 & 0.24 & 0.28 & 0.27 & 0.20 & 0.19 & 0.00 & 0.00 & 0.00 & 0.01 & 0.00 & 0.00 \\
\hline Tfe 3 & 0.08 & 0.00 & 0.00 & 0.00 & 0.00 & 0.00 & 0.00 & 0.00 & 0.00 & 0.00 & 0.00 & 0.00 \\
\hline T-site & 8.00 & 8.00 & 8.00 & 8.00 & 8.00 & 8.00 & 8.08 & 8.07 & 8.03 & 8.00 & 8.05 & 8.01 \\
\hline $\mathrm{Cal}$ & 0.00 & 0.04 & 0.05 & 0.10 & 0.14 & 0.15 & 0.24 & 0.25 & 0.24 & 0.07 & 0.06 & 0.03 \\
\hline $\mathrm{Cfe}^{3}$ & 1.74 & 1.97 & 1.95 & 1.80 & 1.81 & 1.73 & 1.09 & 1.07 & 1.19 & 1.88 & 1.80 & 1.87 \\
\hline Cti & 0.20 & 0.02 & 0.03 & 0.05 & 0.04 & 0.04 & 0.02 & 0.02 & 0.02 & 0.02 & 0.02 & 0.01 \\
\hline $\mathrm{CMg}$ & 0.11 & 0.11 & 0.15 & 0.14 & 0.13 & 0.13 & 0.48 & 0.46 & 0.45 & 0.19 & 0.14 & 0.08 \\
\hline $\mathrm{Cfe}^{2}$ & 2.80 & 2.75 & 2.72 & 2.80 & 2.79 & 2.80 & 2.98 & 3.01 & 2.96 & 2.81 & 2.90 & 2.94 \\
\hline CMn & 0.15 & 0.11 & 0.10 & 0.11 & 0.11 & 0.14 & 0.10 & 0.11 & 0.12 & 0.04 & 0.04 & 0.06 \\
\hline Cca & 0.00 & 0.00 & 0.00 & 0.00 & 0.00 & 0.00 & 0.08 & 0.07 & 0.03 & 0.00 & 0.01 & 0.01 \\
\hline C-site & 5.00 & 5.00 & 5.00 & 5.00 & 5.00 & 5.00 & 5.00 & 5.00 & 5.00 & 5.00 & 4.96 & 5.00 \\
\hline Bca & 0.17 & 0.12 & 0.14 & 0.16 & 0.14 & 0.15 & 0.05 & 0.05 & 0.11 & 0.01 & 0.00 & 0.01 \\
\hline Bna & 1.79 & 1.80 & 1.79 & 1.78 & 1.75 & 1.76 & 1.95 & 1.95 & 1.89 & 1.99 & 1.97 & 1.98 \\
\hline B-site & 1.96 & 1.92 & 1.93 & 1.93 & 1.89 & 1.91 & 2.00 & 2.00 & 2.00 & 2.00 & 1.97 & 1.99 \\
\hline Ana & 0.00 & 0.00 & 0.00 & 0.00 & 0.00 & 0.00 & 0.07 & 0.12 & 0.13 & 0.00 & 0.00 & 0.00 \\
\hline $\mathrm{AK}$ & 0.20 & 0.14 & 0.15 & 0.19 & 0.16 & 0.16 & 0.16 & 0.17 & 0.17 & 0.03 & 0.02 & 0.04 \\
\hline A-site & 0.20 & 0.14 & 0.15 & 0.19 & 0.16 & 0.16 & 0.23 & 0.28 & 0.30 & 0.03 & 0.02 & 0.04 \\
\hline Total cat. & 15.16 & 15.06 & 15.07 & 15.12 & 15.05 & 15.07 & 15.32 & 15.36 & 15.33 & 15.02 & 15.00 & 15.04 \\
\hline
\end{tabular}

were performed by Conceição (1990) and Plá Cid (1994). New data on biotite, pyroxene, and amphibole are presented below. The analyses were performed in the laboratories of Universidade Federal da Bahia (UFBA) and Universidade Federal do Rio Grande do Sul (UFRGS), Brazil, and Universitè de Paris-Sud, Orsay-France. Representative analyses of the minerals are shown in Table 2-4.

\subsection{Mica}

According to the revision of mica classification (Rieder et al, 1998), the Serra do Meio micas plot in the biotite field, at high $\mathrm{FeO} /(\mathrm{FeO}+\mathrm{MgO})$ ratios $(0.64-0.95)$ and close to the annite end member (Fig. 6).

The metamorphic biotite in contact with magnetite phenoblasts, indicates a probable recrystallisation under the influence of metamorphic Fe-bearing fluids (Plá Cid et al., 1995). Chemically, these grains are characterised by lower $\mathrm{Fe} /(\mathrm{Fe}+\mathrm{Mg})$ ratios of about 0.7 that decrease towards the rims (Fig. 7). A similar decrease in the (Fe/
$\mathrm{Fe}+\mathrm{Mg}$ ) ratio in biotite is described by Czamanske and Wones (1973) for the Finnmarka Complex, Oslo — Norway and is interpreted as reflecting increasing $\mathrm{fO}_{2}$ conditions during magmatic evolution. Metamorphic micas have cationic contents in octahedral sites similar to those present in magmatic biotites from peralkaline granites (Fig. 8). Thus, it is inferred that metamorphism during the Brasiliano event promoted changes in the $\mathrm{Fe} / \mathrm{Mg}$ ratios under higher $\mathrm{fO}_{2}$ conditions, but cation filling in the octahedral site was preserved.

Magmatic biotites evolve from annite to siderophylite (Fig. 6). As seen in Fig. 8, the evolution annite $\rightarrow$ siderophylite promotes a decrease in octahedral $\mathrm{Fe}^{+2}, \mathrm{Mn}$, and $\mathrm{Mg}$, and an increase in ${ }^{\mathrm{VI}} \mathrm{Al}$. The biotites in metaluminous granites are richer in ${ }^{\mathrm{VI}} \mathrm{Al}$ (Fig. 8) and ${ }^{\mathrm{IV}} \mathrm{Al}$ (Table 2) suggesting that the magma composition controls the biotite chemistry. The highest $\mathrm{Fe} /(\mathrm{Fe}+\mathrm{Mg})$ ratio is observed in biotites of metaluminous granites, with slightly lower values in the peralkaline types (Fig. 6). The lack of other mafic phases in metaluminous granites, can explain the high 
Table 4

Representative analyses of pyroxenes from Serra do Meio suite. Samples identification is the same observed in Figs. 12 and $17 \mathrm{~b}$

\begin{tabular}{|c|c|c|c|c|c|c|c|c|c|c|c|c|}
\hline Sample & $\begin{array}{l}\text { ppb78A } \\
\text { II }\end{array}$ & $\begin{array}{l}\text { ppb78A } \\
\text { III }\end{array}$ & $\begin{array}{l}\text { ppb78A } \\
\text { V }\end{array}$ & $\begin{array}{l}\text { ppb78A } \\
\text { VI }\end{array}$ & $\begin{array}{l}\text { ppb78A } \\
\text { IX }\end{array}$ & $\begin{array}{l}\text { ppb78A } \\
\text { X }\end{array}$ & $\begin{array}{l}\text { ppb78A } \\
\text { XI }\end{array}$ & $\begin{array}{l}\text { ppb78A } \\
\mathrm{a}\end{array}$ & $\begin{array}{l}\text { ppb78A } \\
\text { b }\end{array}$ & $\begin{array}{l}\text { ppb78A } \\
\text { c }\end{array}$ & $\begin{array}{l}\text { ppb78A } \\
\text { d }\end{array}$ & $\begin{array}{l}\text { ppb78A } \\
\mathrm{e}\end{array}$ \\
\hline $\mathrm{SiO}_{2}$ & 51.52 & 52.86 & 52.26 & 51.47 & 52.15 & 53.02 & 52.88 & 53.39 & 53.34 & 53.56 & 53.83 & 53.68 \\
\hline $\mathrm{Al}_{2} \mathrm{O}_{3}$ & 0.34 & 1.49 & 0.93 & 0.95 & 0.24 & 1.17 & 0.30 & 0.22 & 0.19 & 0.31 & 0.24 & 1.72 \\
\hline $\mathrm{TiO}_{2}$ & 1.52 & 0.11 & 0.89 & 1.42 & 1.36 & 0.15 & 1.54 & 5.25 & 3.64 & 1.25 & 2.04 & 0.10 \\
\hline $\mathrm{FeO}$ & 11.23 & 10.34 & 9.81 & 13.24 & 14.90 & 12.12 & 13.01 & 24.95 & 25.08 & 28.10 & 27.55 & 27.18 \\
\hline $\mathrm{Fe}_{2} \mathrm{O}_{3}$ & 20.08 & 20.80 & 21.17 & 16.27 & 16.66 & 19.50 & 18.24 & 0.00 & 0.00 & 0.00 & 0.00 & 0.00 \\
\hline $\mathrm{MnO}$ & 0.36 & 0.44 & 0.41 & 0.72 & 0.46 & 0.38 & 0.36 & 0.00 & 0.00 & 0.00 & 0.00 & 0.00 \\
\hline $\mathrm{CaO}$ & 4.36 & 3.47 & 3.03 & 5.68 & 6.09 & 3.72 & 3.60 & 2.08 & 1.50 & 0.77 & 0.92 & 3.12 \\
\hline $\mathrm{MgO}$ & 0.04 & 0.14 & 0.14 & 0.11 & 0.01 & 0.16 & 0.02 & 0.01 & 0.00 & 0.02 & 0.00 & 0.13 \\
\hline $\mathrm{Na}_{2} \mathrm{O}$ & 9.85 & 10.29 & 10.55 & 8.92 & 8.71 & 9.91 & 10.05 & 12.08 & 12.59 & 13.07 & 13.01 & 11.67 \\
\hline $\mathrm{K}_{2} \mathrm{O}$ & 0.02 & 0.03 & 0.01 & 0.00 & 0.00 & 0.00 & 0.00 & 0.00 & 0.02 & 0.00 & 0.00 & 0.02 \\
\hline Total & 99.32 & 99.98 & 99.20 & 98.76 & 100.58 & 100.14 & 99.99 & 98.10 & 96.39 & 97.14 & 97.61 & 97.92 \\
\hline TSi & 2.03 & 2.05 & 2.04 & 2.04 & 2.04 & 2.06 & 2.06 & 2.05 & 2.07 & 2.05 & 2.05 & 2.05 \\
\hline Tal & 0.00 & 0.00 & 0.00 & 0.00 & 0.00 & 0.00 & 0.00 & 0.00 & 0.00 & 0.00 & 0.00 & 0.00 \\
\hline Tfe3 & 0.00 & 0.00 & 0.00 & 0.00 & 0.00 & 0.00 & 0.00 & 0.00 & 0.00 & 0.00 & 0.00 & 0.00 \\
\hline M1Al & 0.02 & 0.07 & 0.04 & 0.04 & 0.01 & 0.05 & 0.01 & 0.01 & 0.01 & 0.01 & 0.01 & 0.08 \\
\hline M1Ti & 0.05 & 0.00 & 0.03 & 0.04 & 0.04 & 0.00 & 0.05 & 0.15 & 0.10 & 0.04 & 0.06 & 0.00 \\
\hline $\mathrm{M} 1 \mathrm{Fe} 3$ & 0.59 & 0.60 & 0.62 & 0.48 & 0.49 & 0.57 & 0.53 & 0.49 & 0.60 & 0.79 & 0.73 & 0.68 \\
\hline $\mathrm{M} 1 \mathrm{Fe} 2$ & 0.35 & 0.32 & 0.30 & 0.43 & 0.46 & 0.37 & 0.41 & 0.32 & 0.22 & 0.11 & 0.15 & 0.19 \\
\hline $\mathrm{M} 1 \mathrm{Mg}$ & 0.00 & 0.01 & 0.01 & 0.01 & 0.00 & 0.01 & 0.00 & 0.00 & 0.00 & 0.00 & 0.00 & 0.01 \\
\hline $\mathrm{M} 2 \mathrm{Mg}$ & 0.00 & 0.00 & 0.00 & 0.00 & 0.00 & 0.00 & 0.00 & 0.00 & 0.00 & 0.00 & 0.00 & 0.00 \\
\hline $\mathrm{M} 2 \mathrm{Fe} 2$ & 0.03 & 0.02 & 0.02 & 0.01 & 0.03 & 0.03 & 0.02 & 0.00 & 0.00 & 0.00 & 0.00 & 0.00 \\
\hline $\mathrm{M} 2 \mathrm{Ca}$ & 0.18 & 0.14 & 0.13 & 0.24 & 0.26 & 0.16 & 0.15 & 0.09 & 0.06 & 0.03 & 0.04 & 0.13 \\
\hline $\mathrm{M} 2 \mathrm{Na}$ & 0.75 & 0.77 & 0.80 & 0.69 & 0.66 & 0.75 & 0.76 & 0.90 & 0.95 & 0.97 & 0.96 & 0.86 \\
\hline M2K & 0.00 & 0.00 & 0.00 & 0.00 & 0.00 & 0.00 & 0.00 & 0.00 & 0.00 & 0.00 & 0.00 & 0.00 \\
\hline Total & 3.99 & 3.99 & 3.99 & 3.98 & 3.99 & 3.99 & 3.99 & 4.00 & 4.00 & 4.00 & 4.00 & 4.00 \\
\hline
\end{tabular}

$\mathrm{FeO} /(\mathrm{FeO}+\mathrm{MgO})$ ratios observed in the micas. By contrast, the lower $\mathrm{FeO} /(\mathrm{FeO}+\mathrm{MgO})$ values in mica from peralkaline rocks are due to crystallisation of coeval Fe-rich pyroxene and amphibole.

Biotite compositional differences are displayed in the Nockolds (1947) diagram (Fig. 9). In agreement with petro-

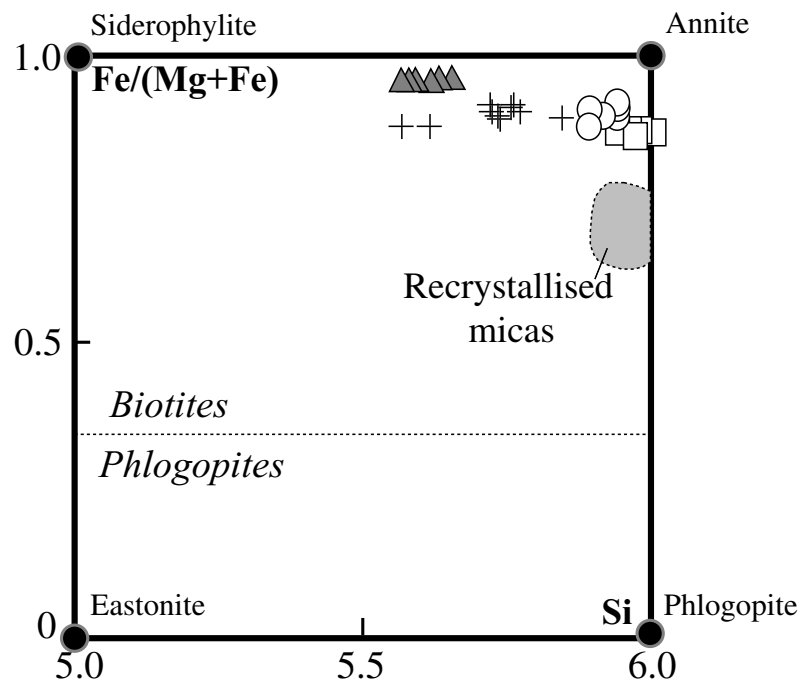

\section{$\triangle$ Biotite granites (NE) $\square$ Slightly peralkaline granites + Biotite magnetite granites $\bigcirc$ Strong peralkaline granites}

Fig. 6. Classification diagram for micas (after Rieder et al., 1998). graphic observations, biotite in metaluminous granites plot in the field of rocks where biotite is the only mafic phase, whereas the biotite from peralkaline rocks fall within the field where biotite is in equilibrium with other mafic minerals. Biotite grains of slightly peralkaline granites are more $\mathrm{Mg}$-rich than those of strongly peralkaline types indicating that biotite in equilibrium with pyroxene is richer in $\mathrm{MgO}$ relative to mica in equilibrium with amphibole (Nockolds, 1947).

Nachit et al. (1985) developed some diagrams for identification of biotites belonging to rocks of different magma series (Fig. 10A). All analyses of the Serra do Meio Suite are typical of biotites crystallised from alkaline magmas and those of metaluminous granites have the highest Al.

Coupled substitutional schemes during magmatic evolution are $\mathrm{Mg}+{ }^{\mathrm{VI}} \mathrm{Al} \rightarrow \mathrm{Fe}^{+2}+\mathrm{Fe}^{+3}$ and ${ }^{\mathrm{IV}} \mathrm{Al}+\mathrm{Fe}^{+3} \rightarrow \mathrm{Si}$ $+\mathrm{Fe}^{+2}$. These correspond to the general scheme $\mathrm{Mg}+$ $\mathrm{Al}_{\text {total }} \rightarrow \mathrm{Si}+2 \mathrm{Fe}^{+2}$ (Fig. 10B) as previously observed by Plá Cid (1994) and originally defined by Czamanske and Wones (1973).

\subsection{Amphibole}

It occurs only in the strongly peralkaline granites. Representative analyses are indicate in Table 3 and belong to the sodic group (Leake, 1978; Leake et al., 1997). The $\mathrm{Ca}+{ }^{\mathrm{IV}} \mathrm{Al}$ vs. $\mathrm{Na}+\mathrm{K}+\mathrm{Si}$ diagram (Giret et al., 1980) indicates that amphibole of the Serra do Meio Suite is either pure 


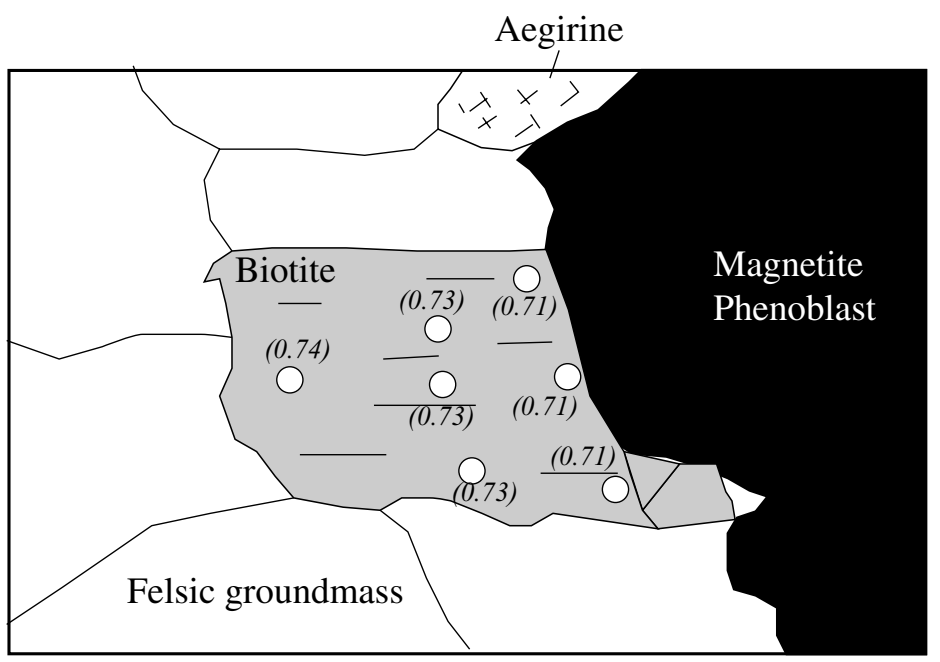

Fig. 7. Textural feature of the slightly peralkaline granite with metamorphic growth of magnetite in contact with biotite. In the biotite crystal are shown some values of the $\mathrm{Fe} /(\mathrm{Mg}+\mathrm{Fe})$ ratio.

riebeckite, or a member of riebeckite-arfvedsonite and riebeckite-winchite solid solutions (Fig. 11A). These evolved compositions reflect the high $\mathrm{SiO}_{2}$-contents of the whole rock which varies between 73 and $74 \mathrm{wt} . \%$. The occurrence of riebeckite-arfvedsonite compositions suggests a late-stage magmatic trend, part of the richterite-arfvedsonite series, defined by Fabriès (1978) and Giret et al. (1980). According to Bowden (1982), riebeckite is a subsolidus mineral produced by reaction between earlier mafic minerals and water-, albite-, and acmite-rich fluids.

Magmatic amphiboles are riebeckite-arfvedsonite and riebeckite-winchite solid solutions with a late-magmatic or subsolidus origin. Riebeckite-arfvedsonite compositions occur in non-oriented subhedral grains whereas riebeckite-

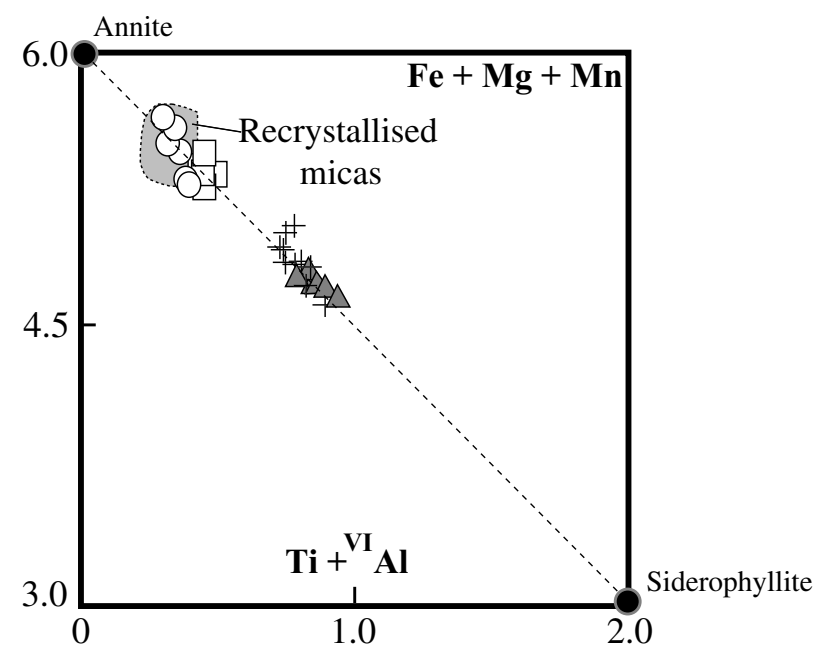

\footnotetext{
$\triangle$ Biotite granites (NE) $\square$ Slightly peralkaline granites + Biotite magnetite granites $\bigcirc$ Strong peralkaline granites
}

Fig. 8. $\mathrm{Fe}+\mathrm{Mg}+\mathrm{Mn}$ vs. $\mathrm{Ti}+{ }^{\mathrm{VI}} \mathrm{Al}$ diagram for Fe-rich micas (after Bonin, 1982). winchite crystals are surrounded by sodic pyroxene (Fig. 12).

Pure riebeckite was considered by Plá Cid (1994) to be a product of recrystallisation. The crystals are subhedral to euhedral porphyroblasts occasionally associated with pure aegirine. They have the highest $\mathrm{Fe}^{+3} / \mathrm{Fe}^{+2}$ ratio $(0.55-0.80)$ whereas in the subsolidus crystals indicate ratios between 0.12 and 0.60 . This is in agreement with the higher $\mathrm{fO}_{2}$ conditions prevailing during metamorphism.

Riebeckite-arfvedsonite and riebeckite-winchite grains have higher $\mathrm{Ca}$-contents than metamorphic grains (Fig. 11) whereas $(\mathrm{Na}+\mathrm{K})$ concentrations are similar in all amphibole types.

The magmatic amphibole (Fig. 13) evolution is controlled by the general substitution: ${ }^{\mathrm{A}} \mathrm{Na}+\mathrm{Fe}^{+2} \rightarrow{ }^{\mathrm{A}} \square+\mathrm{Fe}^{+3}$ (Fabriès, 1978). In the same diagram, the metamorphic amphiboles represent a group with higher values of ${ }^{\mathrm{A}} \square+\mathrm{Fe}^{+3}$. The recrystallised grains have a fill rate in the A-site below $10 \%$, whereas magmatic crystals can reach $37 \%$. This low fill rate in the A-site, compared to more than $90 \%$ of the B-site filled by $\mathrm{Na}$, confirms the riebeckite pure end-member composition (Miyashiro, 1957; Boyd, 1959) for metamorphic amphiboles.

\subsection{Pyroxene}

Pyroxenes are only present in peralkaline rocks as suggested by Neumann (1976) who showed that sodic pyroxenes appear only in magmas with an agpaitic character $(\mathrm{Na}+\mathrm{K} / \mathrm{Al})$ higher than 1 . According to the IMA nomenclature proposition (Morimoto, 1988), they are aegirineaugite and aegirine (Fig. 14). Representative analyses are listed in Table 4.

In the slightly peralkaline granites, pyroxene is aegirineaugite and pure aegirine (Fig. 14), with a compositional gap between both pyroxenes. The aegirine-augite grains are interpreted as preserved magmatic pyroxene whereas pure 

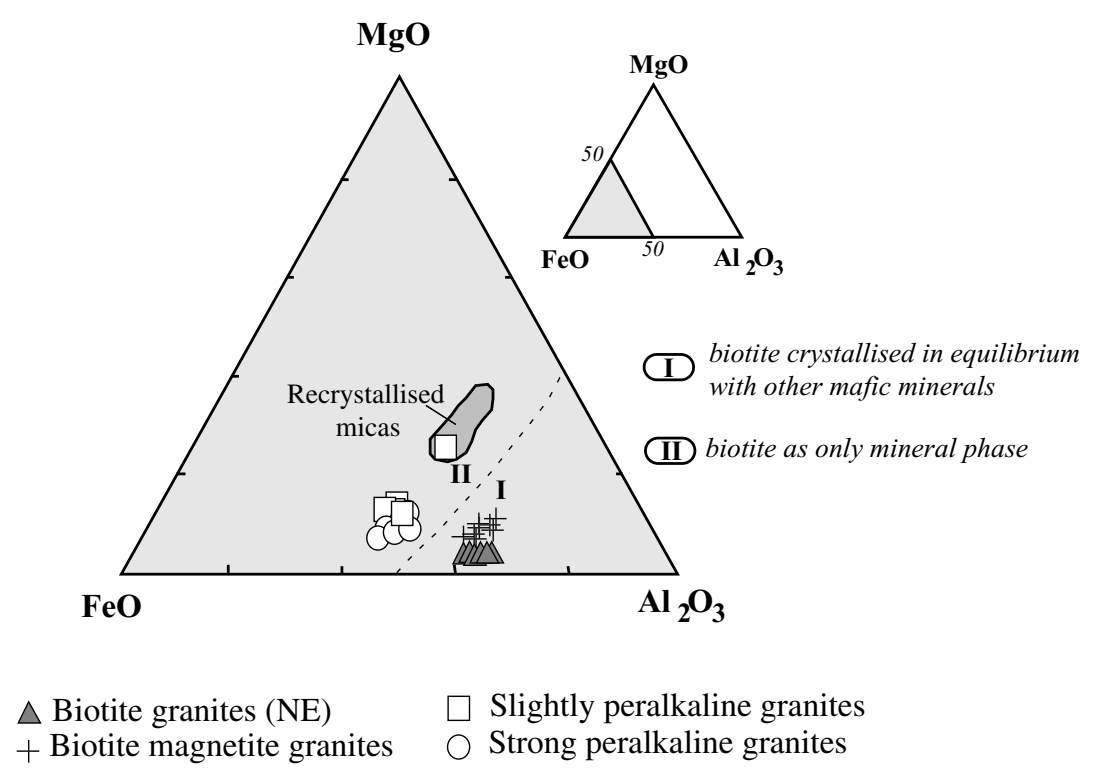

$\square$ Slightly peralkaline granites

Strong peralkaline granites

Fig. 9. Triangular diagram after Nockolds (1947) for discrimination of biotite coexisting with other mafic minerals (olivine, pyroxene, amphibole), and biotite as the only mafic mineral.

aegirine is formed through metamorphic recrystallisation of aegirine-augite.

Magmatic pyroxene crystals from strongly peralkaline granites display compositional zoning varying from aegirineaugite to aegirine (Fig. 14). These grains are characterised by Ti-rich zones, containing up to $15 \%$ of the $\mathrm{Na}_{2} \mathrm{FeTiSi}_{4} \mathrm{O}_{12}$ NAT (neptunite) molecule ( $\mathrm{TiO}_{2}$ up to $5.25 \mathrm{wt} . \%$ ), with very low concentrations of jadeite component (Fig. 14). This titanium-rich composition is probably controlled by $\mathrm{TiO}_{2}$ contents in the magma, since the strongly peralkaline granites are richer in $\mathrm{TiO}_{2}$ than the other types (Fig. 2C). According to Nielsen (1979), Ti-aegirine crystallises under liquidus conditions down to temperatures of $600^{\circ} \mathrm{C}$ Ferguson (1977). Larsen (1976) and Nielsen (1979) argued that Ti-Fe ${ }^{+2}$-pyroxene is produced under low $\mathrm{fO}_{2}$ conditions.

The zoned pyroxenes are either subhedral grains or occur along the margin of winchite-riebeckite subhedral phenocrysts. The petrographic and electron probe data indicate a late magmatic or subsolidus crystallisation order: riebeckite-winchite $\rightarrow$ Ti-aegirine-augite $\rightarrow$ Ti-aegirine. This paragenesis, as inferred by Ferguson (1978), Bonin (1980) and Bonin and Giret (1985), suggests that Ti-bearing aegirine crystallises after calcic and sodic amphiboles. In the strongly peralkaline granites, recrystallised grains have aegirine and aegirine-augite compositions with $\mathrm{TiO}_{2}$ contents lower than 0.2 wt. $\%$ and higher $\mathrm{Al}_{2} \mathrm{O}_{3}$ concentrations (analyses $\mathrm{x}$ and e - Table 4). This $\mathrm{TiO}_{2}$-loss in pyroxene during deformation produced the metamorphic paragenesis aegirine + titanite + (astrophyllite or aenigmatite). In the samples without Ti-pyroxene is not observed any Ti-bearing mineral, and the pyroxene composition ranges between aegirine-augite and aegirine (Fig. 14). In this case, the magmatic and metamorphic aegirine crystals are chemically very similar.
The pyroxenes plot near the acmite apex (Fig. 15A), within the peralkaline silica-saturated field (Bonin and Giret, 1985). As noted by Neumann (1976) and Bonin and Giret (1985), aegirine compositions are compatible with a high agpaitic index and high silica activity in the magma.

The substitutional schemes are described by the oxidising trends $\mathrm{Ca}+\mathrm{Fe}^{+2} \rightarrow \mathrm{Na}+\mathrm{Fe}^{+3}$ (Fig. 15B) and $\mathrm{Ca}+\mathrm{Ti}+$ $\mathrm{Fe}^{+2} \rightarrow \mathrm{Na}+2 \mathrm{Fe}^{+3}$ (Fig. 15C), in magmatic and metamorphic grains. Similar substitutional schemes were reported by Giret et al. (1980) and Bonin and Giret (1985). The $\mathrm{Fe}^{+3} / \mathrm{Fe}^{+2}$ vs. Ti diagram shows magmatic crystallisation of Ti-pyroxene under lower and constant $\mathrm{fO}_{2}$-conditions, when compared to Ti-free pyroxene from strongly peralkaline granites (Fig. 16). In the slightly peralkaline granites, a dramatic increase in $\mathrm{Fe}^{+3} / \mathrm{Fe}^{+2}$ ratios is observed, with the highest values corresponding to pure aegirine analyses (Fig. 16), confirming the high $\mathrm{fO}_{2^{-}}$ conditions during metamorphism.

\section{Trace and rare earth elements in pyroxene and amphibole}

$\mathrm{Sc}, \mathrm{V}, \mathrm{Sr}, \mathrm{Ba}, \mathrm{Y}, \mathrm{Nb}, \mathrm{Hf}$, and REE in pyroxene and amphibole grains were analysed using an ion microprobe CAMECA-IMS 3F, at the laboratory of the CRPG-Centre de Recherches Pétrographiques et Géochimiques, NancyFrance. Analytical data are presented in Table 5.

\subsection{Pyroxene}

Crystals of three different samples were analysed: $(i)$ PPB-78A, strongly peralkaline granite, zoned Ti-aegirine; (ii) GA-46, strongly peralkaline granites, interstitial aegirine oriented along foliation, and, (iii) CL-87, slightly 


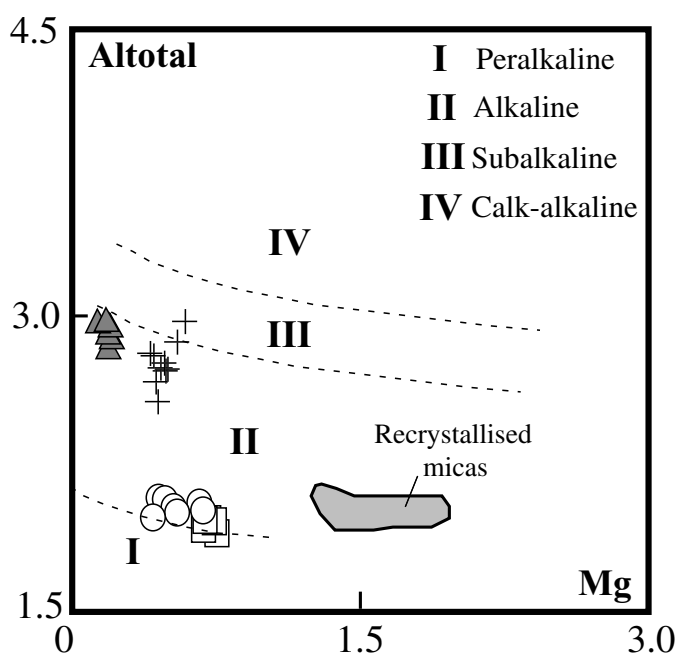

(A)

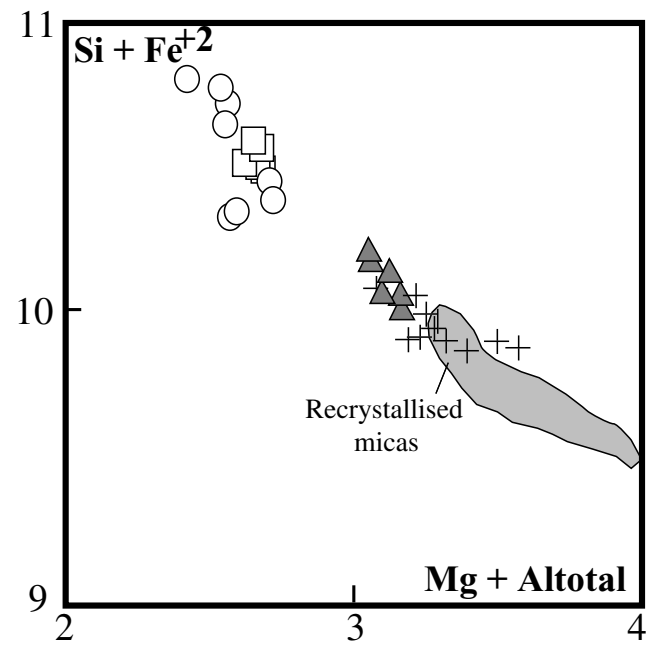

(B)

\section{$\triangle$ Biotite granites (NE) + Biotite magnetite granites}

Slightly peralkaline granites Strong peralkaline granites

Fig. 10. Al vs. Mg diagram of Nachit et al. (1985), showing micas of the different magmatic series (A). $\mathrm{Si}+\mathrm{Fe}^{+2} \mathrm{vs.} \mathrm{Mg}+\mathrm{Al}$ diagram showing biotite evolution in the Serra do Meio suite (B).

peralkaline granites, subhedral grains of millimetre-size irregular agglomerations.

As noted by Jacobson et al. (1958), Ernst (1962), Larsen (1976), Neumann (1976), Bonin (1980) and Mitchell (1990), mafic minerals from the sodic series crystallised late, and do not reflect the original liquid composition. Previous studies on trace and REE contents in pyroxene from peralkaline systems are rare and generally restricted to early crystallised phenocrysts (Larsen, 1979; Vannucci et al., 1991; Dorais and Floss, 1992). Aegirine grains in peralkaline suites were analysed by Shearer et al. (1989) and Shearer and Larsen (1994) in the Ilímaussaq complex, South Greenland.

In the strongly peralkaline granites, Ti-aegirine zoned crystals of sample PPB-78A contain a Ti-rich core $\left(\mathrm{TiO}_{2}\right.$ - 3.5-5.2 wt.\%), with concentrations along the rims varying from 1.2 to $2.0 \mathrm{wt} . \%$. The lowest concentrations $(<0.1 \mathrm{wt} . \%)$ occur along the outer recrystallised portion. Ti zonation is mirrored by REE where the Ti-rich core has the highest trace element and REE concentration (Fig. 17A) whereas the lowest values occur in the recrystallised rim.

The REE patterns of Ti-zoned pyroxene were normalised to the chondritic values of Evensen et al. (1978; Fig. 17A). The core is enriched in all rare earth elements, particularly in the intermediate group, with a slight $\mathrm{Eu}-$ negative anomaly (Fig. 17A). The high REE-contents of these pyroxenes confirms their incompatible behaviour in peralkaline oversaturated magmas. The rims, with 

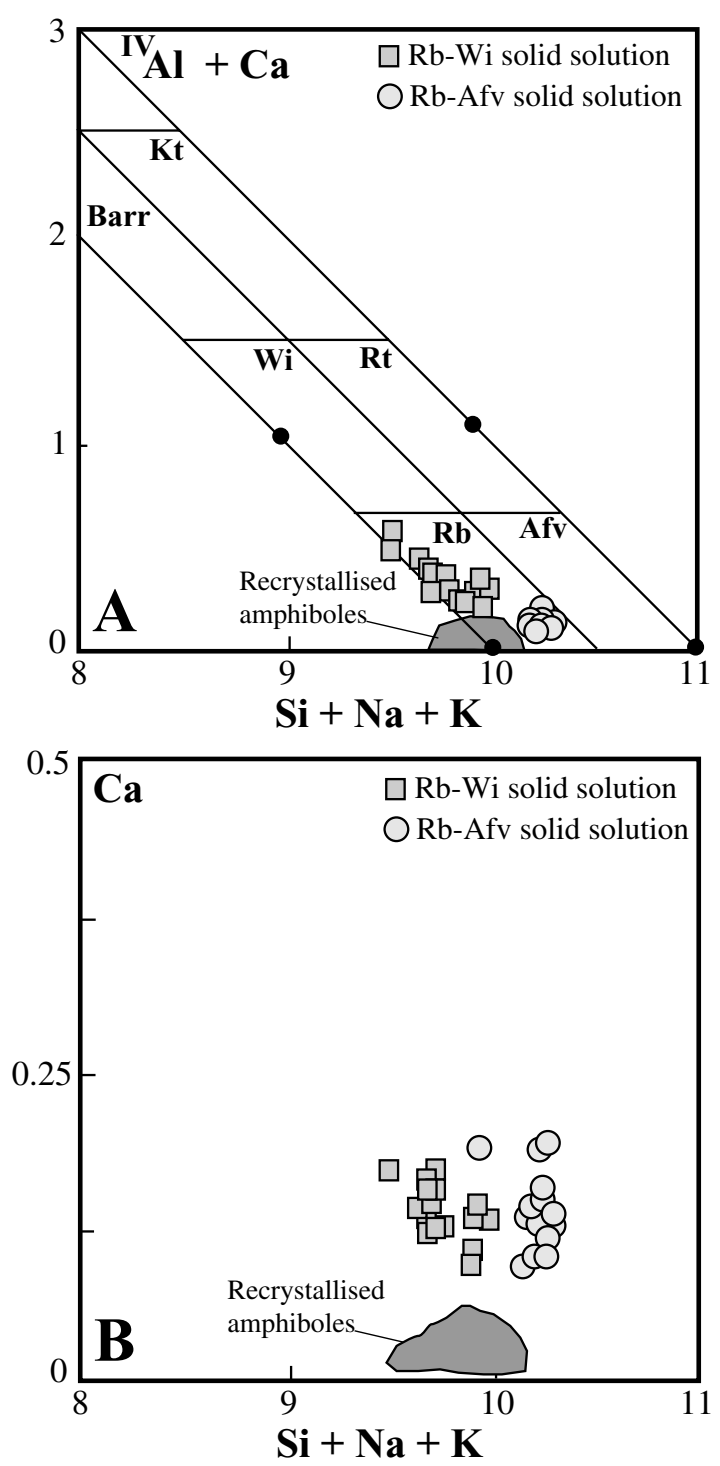

Fig. 11. Classification diagram for alkali-amphiboles (Giret et al., 1980). Katophorite (Kt), Barroisite (Barr), Winchite (Wi), Richterite (Rt), Arfvedsonite (Afv), and Riebeckite (Rb). (A) The filled circles indicate the composition of the end members. (B) $\mathrm{Ca}$ vs. $\mathrm{Si}+\mathrm{K}+\mathrm{Na}$ diagram discriminates the $\mathrm{Ca}$ contents in magmatic and metamorphic grains.

intermediate Ti-concentrations are depleted in intermediate rare earth elements (Fig. 17A) due to strong REEpartitioning in the Ti-rich core. The REE patterns of these rims are similar to those obtained by Larsen (1979) in hedenbergite and by Shearer and Larsen (1994) in aegirine (Fig. 17A), confirming their probable magmatic origin. The recrystallised rim has the lowest REE-content, mainly due to a decrease in the amount of light rare earth elements (Fig. 17A). Thus, metamorphism of Ti-aegirine resulted in the loss of $\mathrm{Ti}$ and light rare earth elements along the rims.

The Ti-aegirine crystal has low concentrations of $\mathrm{Ba}$ (1-7 ppm), Sr (7.2-23.4 ppm) and V (8.7-18.3 ppm) similar to those analysed by Shearer and Larsen (1994). The low concentrations reflect the very low abundance of these elements in alkaline oversaturated liquids. On the contrary, Plá Cid et al. (1999) found that ultrapotassic syenites contain $\mathrm{Sr}$ and V-rich aegirine-augite crystals reflecting their early crystallisation from $\mathrm{Sr}$ and $\mathrm{V}$-rich liquid.

Ti-aegirine from strongly peralkaline granites is enriched in $\mathrm{Y}(8-340 \mathrm{ppm}), \mathrm{Nb}(5-613 \mathrm{ppm})$ and $\mathrm{Hf}(6-85 \mathrm{ppm})$ showing a positive correlation among these elements (Fig. $17 b)$. In figure $17 \mathrm{C}$, where $\mathrm{Y}_{\text {aegirine }} / \mathrm{Y}_{\text {rock }}$ vs. $N \mathrm{Nb}_{\text {aegirine }} / \mathrm{Nb}_{\text {rock }}$ ratios are plotted, the same positive correlation is observed suggesting that $\mathrm{Y}$ and $\mathrm{Nb}$ contents in Ti-aegirine are directly correlated to the concentrations in the liquid. The initial crystallisation of this pyroxene is marked by strong partitioning of $\mathrm{Y}, \mathrm{Nb}, \mathrm{Hf}$, and REE which depletes the residual magmatic liquid in these elements and results in lower concentrations along the rims (Fig. 17b). The metamorphic rim is therefore characterised by $\mathrm{Nb}$ and $\mathrm{Hf}$ depletion, and $\mathrm{Y}$ enrichment (Fig. 17B and C).

The REE contents of recrystallised aegirine in sample GA-46 are enriched nearly 10 times the chondritic values (Fig. 17A). Their $\Sigma$ REE contents show a strong depletion relative to Ti-pyroxenes. The REE patterns are roughly flat and very different from those reported by Larsen (1979) and Shearer and Larsen (1994). These uncommon patterns probably reflect metamorphic equilibration and not magmatic concentrations.

The whole-rock concentrations of $\mathrm{Nb}, \mathrm{Y}$, and $\mathrm{Hf}$ in sample GA-46 are lower than in the slightly peralkaline granites (sample - CL-87; Table 1), although the partition coefficients for mineral/rock are similar (Fig. 17C). In the strongly peralkaline granites, incompatible elements are preferentially concentrated in Na-pyroxenes whereas in the slightly peralkaline granites, these elements are also partitioned into other minerals. Therefore, the rare earth elements in peralkaline systems are more easily mobilised by fluids during metamorphism than other trace elements.

In the slightly peralkaline granites the subhedral sodic pyroxene from irregular agglomerations has lower REE contents than pyroxene grains from strongly peralkaline granites (Fig. 17A). REE abundances range between 10 and $44 \mathrm{ppm}$, and the shape of the patterns is similar to those of Ti-rich core pyroxene of strongly peralkaline granites. The patterns, as well as the textural features, reflect their magmatic origin.

$\mathrm{Ba}, \mathrm{Sr}$ and $\mathrm{V}$ contents are very depleted in pyroxene grains of this facies and normally lower than in Ti-aegirine from strongly peralkaline granites (Table 5). The $\mathrm{Nb}_{\text {aegirine }} /$ $\mathrm{Nb}_{\text {rock }}$ and $\mathrm{Y}_{\text {aegirine }} / \mathrm{Y}_{\text {rock }}$ ratios are also lower than those observed in Ti-aegirine and show the same positive correlation between concentrations in mineral and rock (Fig. 17C). The slightly peralkaline granites are richer in $\mathrm{Nb}$-, $\mathrm{Y}-$, and $\mathrm{Hf}$ relative to the strongly peralkaline varieties, and the mineral/rock elemental ratios are lower. This is probably due to accommodation of these elements by other minerals prior to late-stage or subsolidus pyroxene crystallisation. 

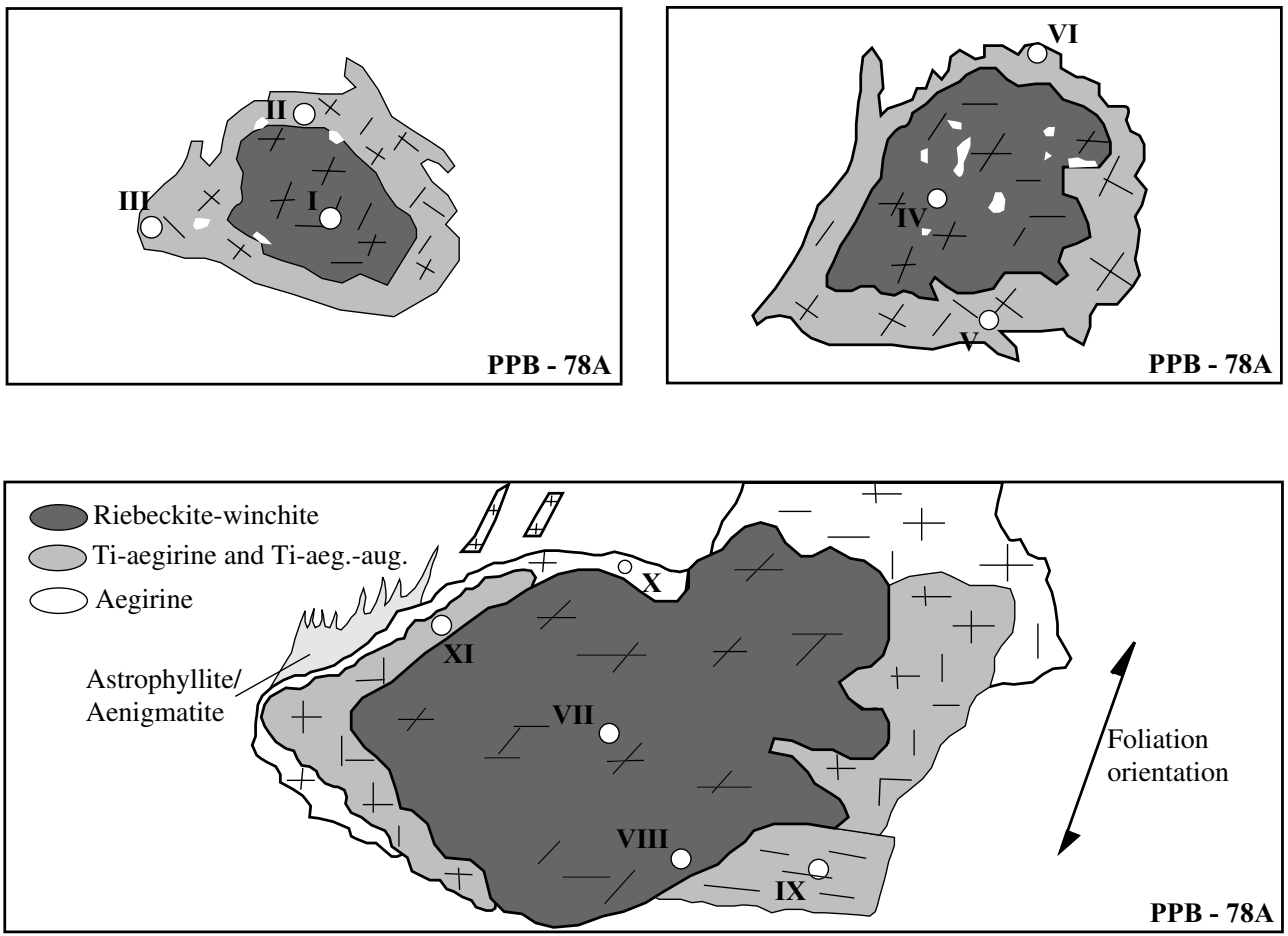

Fig. 12. Textural features of the strongly peralkaline granites showing the crystallisation order riebeckite-winchite, Ti-Na-pyroxene, and the metamorphic paragenesis-aegirine + astrophyllite/aenigmatite. The Roman numerals indicate the analyses observed in Tables 2-4.

\subsection{Amphibole}

The trace and rare earth element analyses for amphiboles are listed in Table 6. In samples PPP-78A and GA-34, amphibole occurs as oriented porphyroblasts with rims of reddish, fibrous, astrophyllite or aenigmatite. In sample GA46 , the analysed grain is interstitial and oriented parallel to the metamorphic foliation.

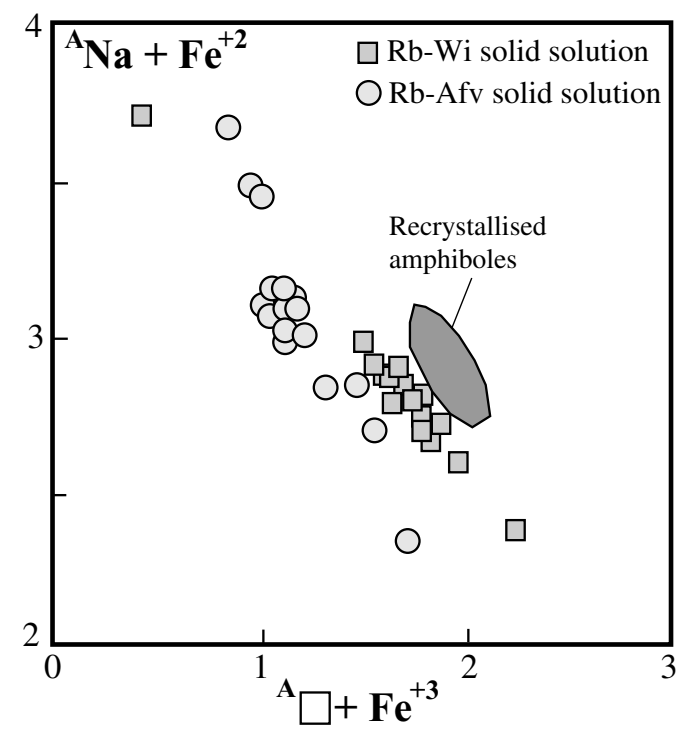

Fig. 13. Substitutional scheme for amphiboles from the Serra do Meio suite.
REE are generally enriched relative to chondritic values (Fig. 18), with absolute concentrations ranging from 2 to $275 \mathrm{ppm}$. These patterns are roughly flat, at about 10 times the chondritic values along with Eu-negative anomalies. The interstitial grains yield the highest concentrations of REE (275 ppm) and show intensive HREE fractionation (Fig. 18).

Compared with pyroxenes, amphiboles yield lower REE concentrations. The recrystallised pyroxenes from sample GA-46 exhibit REE patterns that are similar to those

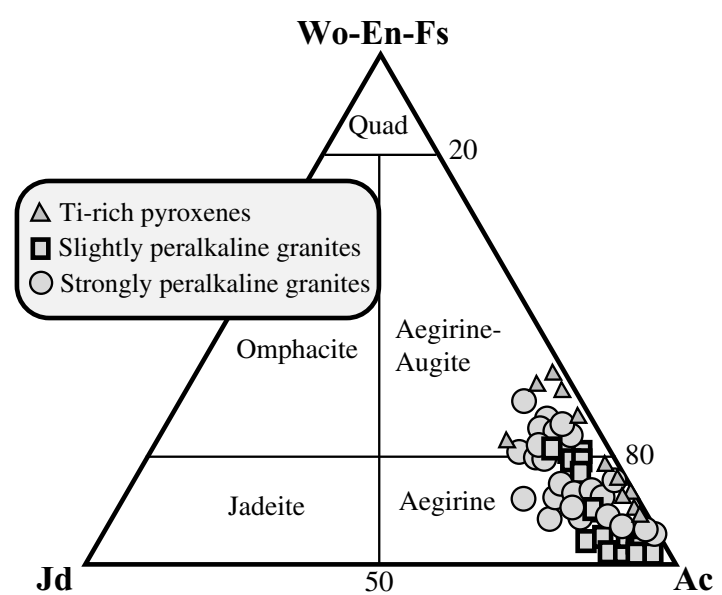

Fig. 14. QUAD-Jd-Ac triangular diagram for classification of sodic pyroxenes (after, Morimoto, 1988). 


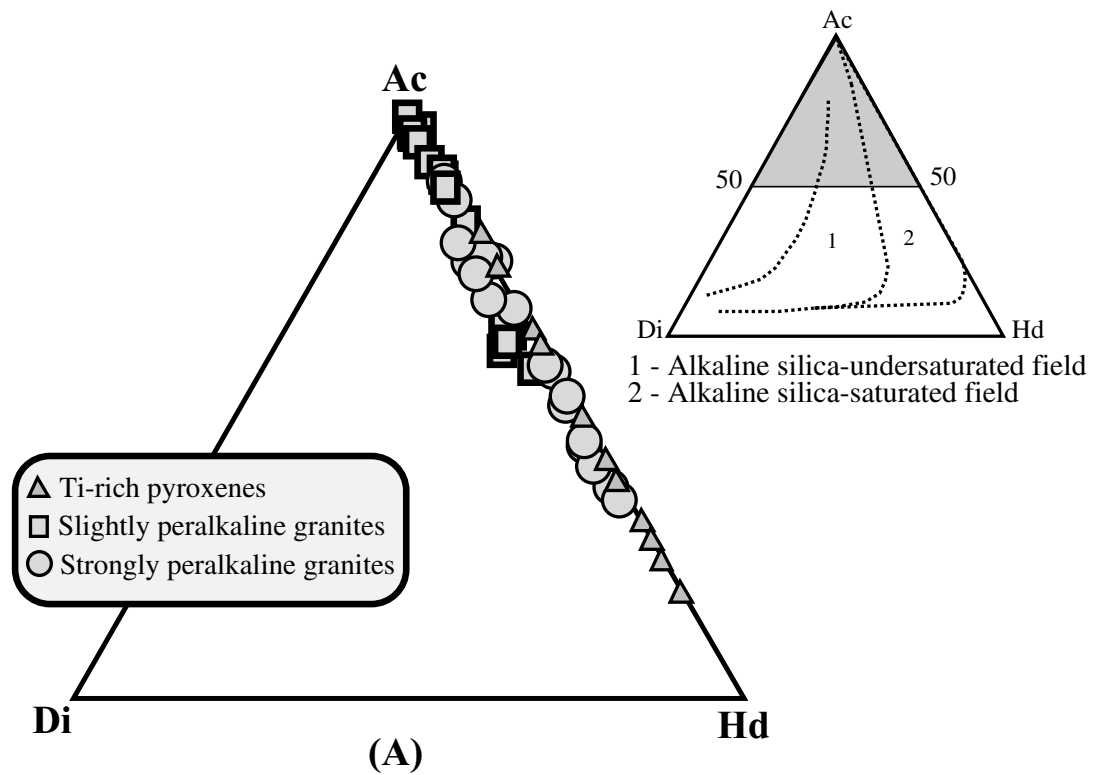

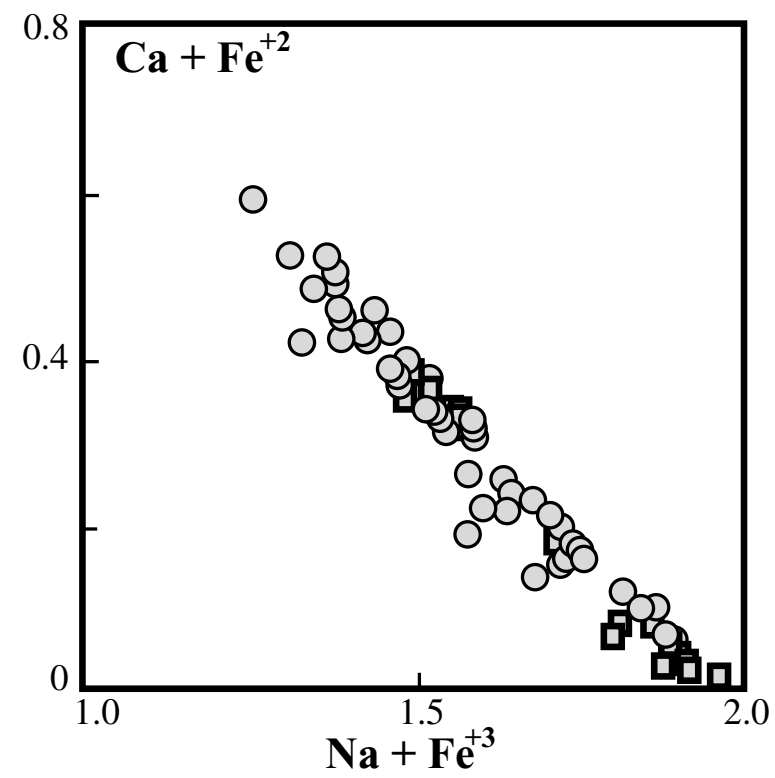

(B)

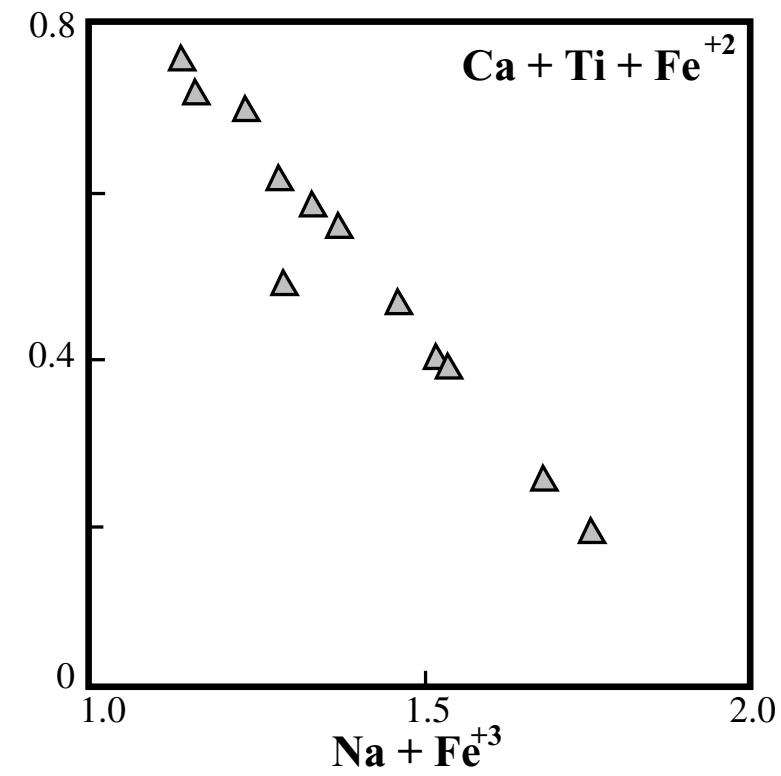

(C)

Fig. 15. (A) Diopside (Di)-Hedenbergite (Hd)-Acmite (Ac) triangular diagram (Bonin and Giret, 1985). (B) Substitutional schemes of the Na- and Na-Ca pyroxenes, and (C) Ti-rich pyroxenes.

observed for amphiboles, although pyroxenes have Ce enrichment relative to La which is not observed in amphiboles. $\mathrm{Sr}, \mathrm{V}$, and $\mathrm{Hf}$ concentrations are depleted relative to pyroxenes, whereas $\mathrm{Ba}$ is enriched (Table 6). $\mathrm{Y}$ and $\mathrm{Nb}$ concentrations are similar in amphiboles and pyroxenes for samples GA-46 and PPB-78A because these Na-rich minerals are the main carriers of these incompatible elements. However, the positive correlation between $\mathrm{Y}, \mathrm{Nb}$, and $\mathrm{Hf}$ in pyroxenes was not observed in amphibole, although the small number of analyses preclude any conclusions.

\section{Final considerations}

\subsection{Magmatic evolution}

Experimental studies have shown that partial melting of crustal sources is unlikely to generate peralkaline granitic magmas (Patiño Douce and Beard, 1996; Dooley and Patiño Douce, 1996). Halliday et al. (1991) argue for intense fractional crystallisation involving substantial volumes of cumulates and claim that generation of high- $\mathrm{Rb} / \mathrm{Sr}$ rhyolites 


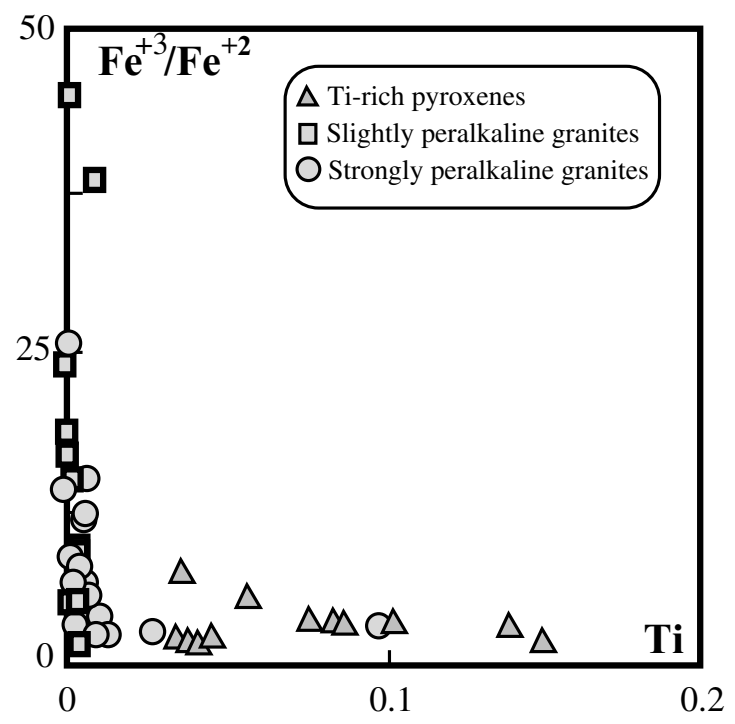

Fig. 16. $\mathrm{Fe}^{+2} / \mathrm{Fe}^{+3}$ and $\mathrm{Ti}$ variations in pyroxenes of the Serra do Meio suite.

cannot be explained by any partial melting models involving the usual crustal sources.

The Serra do Meio suite is characterised by enrichment in incompatible elements such as $\mathrm{Zr}, \mathrm{Nb}, \mathrm{Y}, \mathrm{Ga}$, and light-REE relative to the average composition of A-type granites (Whalen et al., 1987) and to the anorogenic Corsican suite (Bonin, 1980, 1988). Plá Cid et al. (1997) noted their derivation from an incompatible-element enriched mantle source. The HFSE enrichment is lower in peralkaline than in metaluminous rocks. Regarding the trace elements variation diagrams (Fig. 3), Ba contents are different for both evolutionary trends, exhibiting a negative anomaly in metaluminous granites which is absent in peralkaline rocks. This feature, as well as the stronger Eu-negative anomaly in metaluminous granites (Fig. 5), suggest strong fractionation of alkali feldspar.

The contrasting HFSE concentrations in the Serra do Meio metaluminous and peralkaline liquids can be explained by their provenance from different original basic melts. They were produced by a small degrees of melting in the mantle during different stages. The parental magma of metaluminous liquids was first extracted and the HFSE-solubility was enhanced by high temperature and high $\mathrm{F}$ contents in the source. This early extraction caused a relative depletion in the source resulting in lower abundance in subsequent magmas generated during a second partial melting event. This hypothesis is supported by the fact that the Serra do Meio metaluminous granites are richer in HFSE and F relative to typical anorogenic metaluminous granites from Nigeria (Bowden and Kinnaird, 1984).

\subsection{Major and trace elements correlation in Ti-aegirine}

REE and $\mathrm{Zr}$ exhibit a negative correlation with $\mathrm{Na} /$ $(\mathrm{Na}+\mathrm{Ca})$ ratios in aegirine (Shearer and Larsen, 1994). The size of the M2-site in hedenbergite (Cameron et al., 1973) and acmite (Clark et al., 1969) is very similar, showing a limited effect on the site capacity to accommodate REE. In this case, REE incorporation is controlled by the optimal charge differences between hedenbergite (1.79) and acmite (1.16; Shearer and Larsen, 1994). In addition, the crystallisation of REE-bearing phases during pyroxene growth would result in a decrease in REE and $\mathrm{Ca}$ in pyroxene (Larsen, 1977, 1979).

In Ti-aegirine of the Serra do Meio suite, the negative correlation between REE and $\mathrm{Na} /(\mathrm{Na}+\mathrm{Ca})$ ratio is observed as well as with other trace elements (Fig. 19).

Table 5

Trace and rare earth elements (in ppm) of pyroxene from Serra do Meio suite. Identification of analyses of sample PPB-78A are the same as Fig. 17

\begin{tabular}{|c|c|c|c|c|c|c|c|c|c|c|c|c|}
\hline Sample & CL-87 & CL-87 & GA-46 & GA-46 & GA-46 & GA-46 & GA-46 & $\begin{array}{l}\text { PPB78A } \\
\text { b }\end{array}$ & $\begin{array}{l}\text { PPB-78A } \\
\mathrm{c}\end{array}$ & $\begin{array}{l}\text { PPB-78A } \\
\text { e }\end{array}$ & $\begin{array}{l}\text { PPB78A } \\
\text { a }\end{array}$ & $\begin{array}{l}\text { PPB-78A } \\
\mathrm{d}\end{array}$ \\
\hline $\mathrm{Sc}$ & 23.14 & 35.17 & 70.48 & 55.05 & 84.64 & 49.11 & 109.63 & 12.24 & 13.61 & 15.92 & 14.86 & 12.04 \\
\hline $\mathrm{V}$ & 11.96 & 22.88 & 27.70 & 14.34 & 27.58 & 14.20 & 31.14 & 12.89 & 9.52 & 8.66 & 18.31 & 9.47 \\
\hline $\mathrm{Sr}$ & 4.14 & 5.70 & 19.35 & 86.60 & 8.39 & 17.11 & 27.37 & 17.57 & 23.35 & 7.19 & 18.00 & 11.58 \\
\hline Y & 5.51 & 34.66 & 12.11 & 8.29 & 2.42 & 7.78 & 11.53 & 21.78 & 19.36 & 39.65 & 340.47 & 8.41 \\
\hline $\mathrm{Nb}$ & 5.80 & 51.53 & 17.54 & 9.91 & 0.34 & 2.70 & 13.00 & 32.23 & 14.21 & 0.62 & 613.39 & 5.33 \\
\hline $\mathrm{Ba}$ & 196.33 & 1.94 & 4.88 & 218.35 & 1.89 & 2.01 & 3.58 & 1.34 & 1.57 & 1.37 & 7.57 & 1.26 \\
\hline $\mathrm{Hf}$ & 0.49 & 3.19 & 1.79 & 1.37 & 1.25 & 0.00 & 1.26 & 19.96 & 33.05 & 0.00 & 85.47 & 6.60 \\
\hline $\mathrm{La}$ & 0.33 & 0.79 & 2.65 & 14.01 & 0.74 & 0.64 & 1.33 & 8.53 & 11.20 & 1.65 & 16.44 & 8.83 \\
\hline $\mathrm{Ce}$ & 0.95 & 6.49 & 22.65 & 16.73 & 0.54 & 3.15 & 10.41 & 42.37 & 70.36 & 4.06 & 139.77 & 49.16 \\
\hline $\operatorname{Pr}$ & 0.32 & 1.35 & 1.03 & 0.98 & 0.21 & 0.26 & 0.58 & 7.77 & 8.25 & 0.68 & 26.63 & 5.47 \\
\hline $\mathrm{Nd}$ & 1.87 & 8.47 & 3.59 & 3.50 & 3.05 & 0.85 & 2.96 & 39.82 & 37.34 & 4.42 & 187.70 & 20.31 \\
\hline $\mathrm{Sm}$ & 1.65 & 4.83 & 0.77 & 3.84 & 1.59 & 0.63 & 1.18 & 9.98 & 8.18 & 3.76 & 92.59 & 3.28 \\
\hline $\mathrm{Eu}$ & 0.65 & 0.84 & 0.05 & 0.86 & 0.00 & 0.14 & 0.29 & 1.35 & 1.29 & 1.72 & 21.12 & 0.69 \\
\hline $\mathrm{Gd}$ & 1.45 & 6.94 & 0.82 & 5.23 & 1.68 & 0.49 & 1.12 & 6.58 & 6.38 & 3.44 & 129.04 & 1.04 \\
\hline Dy & 1.21 & 5.84 & 0.94 & 3.60 & 1.90 & 1.00 & 1.57 & 4.32 & 4.29 & 6.76 & 111.42 & 1.27 \\
\hline $\mathrm{Er}$ & 1.06 & 3.90 & 0.61 & 1.11 & 1.78 & 0.55 & 0.79 & 5.35 & 3.74 & 2.73 & 66.69 & 1.05 \\
\hline $\mathrm{Yb}$ & 1.06 & 3.58 & 0.89 & 1.63 & 0.65 & 1.19 & 1.64 & 17.81 & 13.29 & 6.22 & 43.07 & 3.35 \\
\hline $\mathrm{Lu}$ & 0.05 & 0.11 & 0.10 & 0.22 & 0.14 & 0.14 & 0.21 & 0.99 & 1.05 & 0.48 & 1.75 & 0.18 \\
\hline
\end{tabular}



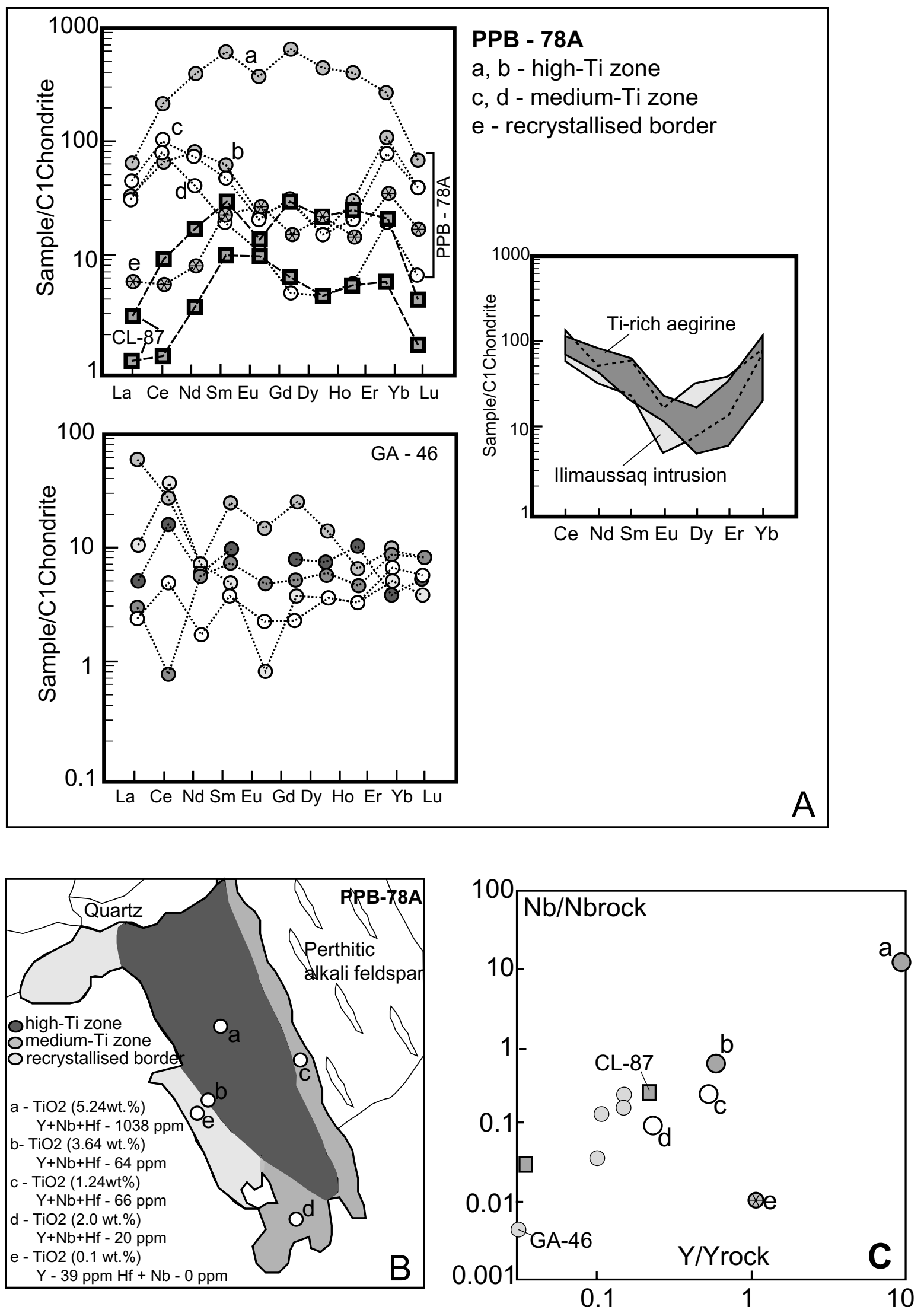

Fig. 17. (A) REE patterns of pyroxenes normalised to the chondritic values of Evensen et al. (1978) and compared with Ti-aegirine from the Ilímaussaq intrusion. (B) Textural feature of Ti-aegirine analysed in sample PPB-78A, with REE patterns observed in A. (C) Nb/Nbrock vs. Y/Yrock diagram for pyroxenes of the Serra do Meio suite. 
Correlation between trace elements such as $\mathrm{Nb}, \mathrm{V}$ and $\mathrm{Y}$, and the major elements occurring in M1 and M2 sites is not clear since the $\mathrm{Na} /(\mathrm{Na}+\mathrm{Ca})$ ratio in these pyroxenes shows a strong correlation with the $\mathrm{Fe}^{+3} / \mathrm{Fe}^{+2}$ ratio and Ti content. It is necessary to verify whether $\mathrm{Nb}, \mathrm{Y}$ and $\mathrm{V}$ are readily incorporated into the M2 site or related to elemental variations in the M1 site. Significant partitioning of trace and rare earth elements between Na-pyroxene and liquid explains the dramatic decrease of these elements in pyroxene rims. Early crystallisation of Na-pyroxene leads to lower concentrations of these elements in the residual liquids, and a strong depletion in the late magmatic rims. In Fig. 19, recrystallised borders show Na loss during metamorphism as evidenced by a decrease in the $\mathrm{Na} /(\mathrm{Na}+\mathrm{Ca})$ ratio accompanied by general depletion of all trace elements.

The slightly peralkaline granites show higher concentrations of $\mathrm{Nb}+\mathrm{Y}$ relative to strongly peralkaline rocks (Table 1). Primary aegirine in the slightly peralkaline granites have lower amounts of $\mathrm{Nb}+\mathrm{Y}$ than Ti-aegirine found in strongly peralkaline rocks. Considering the higher $\mathrm{F}$ contents of the slightly peralkaline granites, $\mathrm{REE}, \mathrm{Y}$, and $\mathrm{Nb}$ are likely to be partitioned between aegirine and exotic F-bearing minerals. In the strong peralkaline rocks, however, these elements are incorporated only into Na-mafic minerals.

\subsection{Recrystallisation conditions}

Plá Cid et al. (2000) noted that recrystallisation of the Serra do Meio suite occurred under high $\mathrm{fO}_{2}$ conditions, within the magnetite stability field, and an upper temperature limit of around $550-600^{\circ} \mathrm{C}$. This is supported by the stability temperature of alkali-amphiboles (Ernst, 1962), and by metamorphic conditions for basement rocks defined

Table 6

Trace and rare earth elements (in ppm) of amphibole crystals from Serra do Meio suite

\begin{tabular}{lrrrrr}
\hline Sample & GA-34 & GA-34 & GA-46 & PPB78A & PPB78A \\
\hline $\mathrm{Sc}$ & 31.45 & 15.74 & 118.74 & 22.47 & 23.00 \\
$\mathrm{~V}$ & 6.83 & 6.56 & 16.88 & 7.23 & 6.71 \\
$\mathrm{Sr}$ & 8.26 & 1.27 & 15.33 & 5.93 & 5.41 \\
$\mathrm{Y}$ & 6.17 & 0.08 & 12.47 & 31.96 & 28.53 \\
$\mathrm{Nb}$ & 17.77 & 1.17 & 6.83 & 10.28 & 35.41 \\
$\mathrm{Ba}$ & 98.58 & 5.02 & 51.86 & 53.54 & 5.62 \\
$\mathrm{Hf}$ & 0.00 & 0.21 & 0.60 & 0.00 & 0.00 \\
$\mathrm{La}$ & 3.56 & 0.17 & 54.94 & 5.87 & 2.58 \\
$\mathrm{Ce}$ & 9.81 & 0.66 & 133.57 & 10.44 & 10.78 \\
$\mathrm{Pr}$ & 1.35 & 0.08 & 16.31 & 2.22 & 1.57 \\
$\mathrm{Nd}$ & 5.91 & 0.56 & 55.04 & 9.05 & 7.66 \\
$\mathrm{Sm}$ & 1.80 & 0.29 & 6.53 & 4.49 & 4.29 \\
$\mathrm{Eu}$ & 0.55 & 0.10 & 0.72 & 0.87 & 0.27 \\
$\mathrm{Gd}$ & 4.08 & 0.36 & 4.96 & 4.48 & 6.10 \\
$\mathrm{Dy}$ & 1.87 & 0.13 & 1.65 & 4.44 & 3.97 \\
$\mathrm{Er}$ & 0.93 & 0.18 & 0.82 & 2.35 & 1.99 \\
$\mathrm{Yb}$ & 2.24 & 0.19 & 0.97 & 7.72 & 4.42 \\
$\mathrm{Lu}$ & 0.15 & 0.06 & 0.07 & 0.27 & 0.14 \\
\hline
\end{tabular}

by Leite (1997), as greenschist to low amphibolite facies in this part of the Riacho do Pontal fold belt.

In metaluminous granites, the higher metamorphic temperatures is illustrated by a similar reaction of annite + quartz $\rightarrow$ alkali feldspar + magnetite + quartz (Eugster and Wones, 1962; Rutherford, 1969), under high $\mathrm{fO}_{2}$ conditions, close to the $\mathrm{Ni}-\mathrm{NiO}$ buffer (Conceição, 1990) and temperature (for $\log \mathrm{fO}_{2}=10^{-18}$ ) estimated at $600^{\circ} \mathrm{C}$ (Fig. 20A). In the presence of Fe-rich fluids (Plá Cid, 1994), the reaction microcline + quartz + Fe-fluids $\rightarrow$ annite + magnetite (Bonin, 1982) occurred at temperatures and $\mathrm{fO}_{2}$ conditions close to those reported by Eugster and Wones (1962). Metamorphism related to the Brasiliano event also caused thermal instability of alkali-feldspar with development of subsolvus albite + microcline paragenesis

In the strongly peralkaline granites, two groups of magmatic amphiboles were identified: (i) riebeckite-winchite grains rimmed by Ti-pyroxene, and (ii) riebeckitearfvedsonite crystals. Amphiboles with such a composition are diagnostic of late-magmatic to subsolidus origin as described by Giret et al. (1980). Bonin (1988) defined a subsolidus trend that is consistent with riebeckitearfvedsonite compositions in the Serra do Meio suite (Fig. 20B). Furthermore, the petrographic relations of riebeckitewinchite grains are suggestive of their earlier crystallisation relative to riebeckite-arfvedsonite crystals.

The oxidising reaction: riebeckite + quartz + fluid $\rightarrow$ aegirine + magnetite + quartz + fluid occurred during metamorphism in the strongly peralkaline rocks (Plá Cid, 1994). The studies of Ernst (1962) on sodic amphiboles stability suggest a temperature below $600^{\circ} \mathrm{C}$ and $\mathrm{fO}_{2}$ conditions within the magnetite field, near to $\mathrm{NiNiO}$ buffer ( $\log \mathrm{fO}_{2}=10^{-20}$ bars, Fig. 20B). These peralkaline granites are also characterised by the presence of magmatic riebeckite-winchite amphibole rimmed by Ti-aegirine and isolated subhedral crystals of Ti-aegirine. Metamorphism of these Ti-pyroxenes produced the syntectonic paragenesis riebeckite + titanite + (aenigmatite

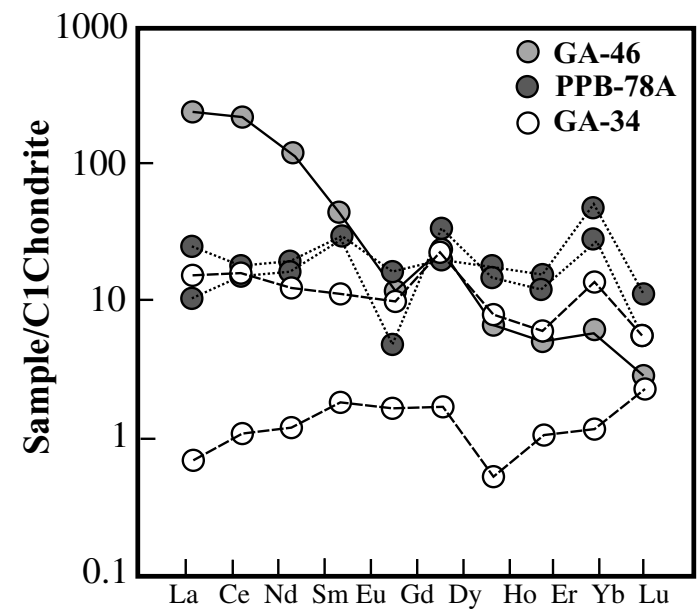

Fig. 18. REE patterns of amphiboles normalised to the chondritic values of Evensen et al. (1978). 

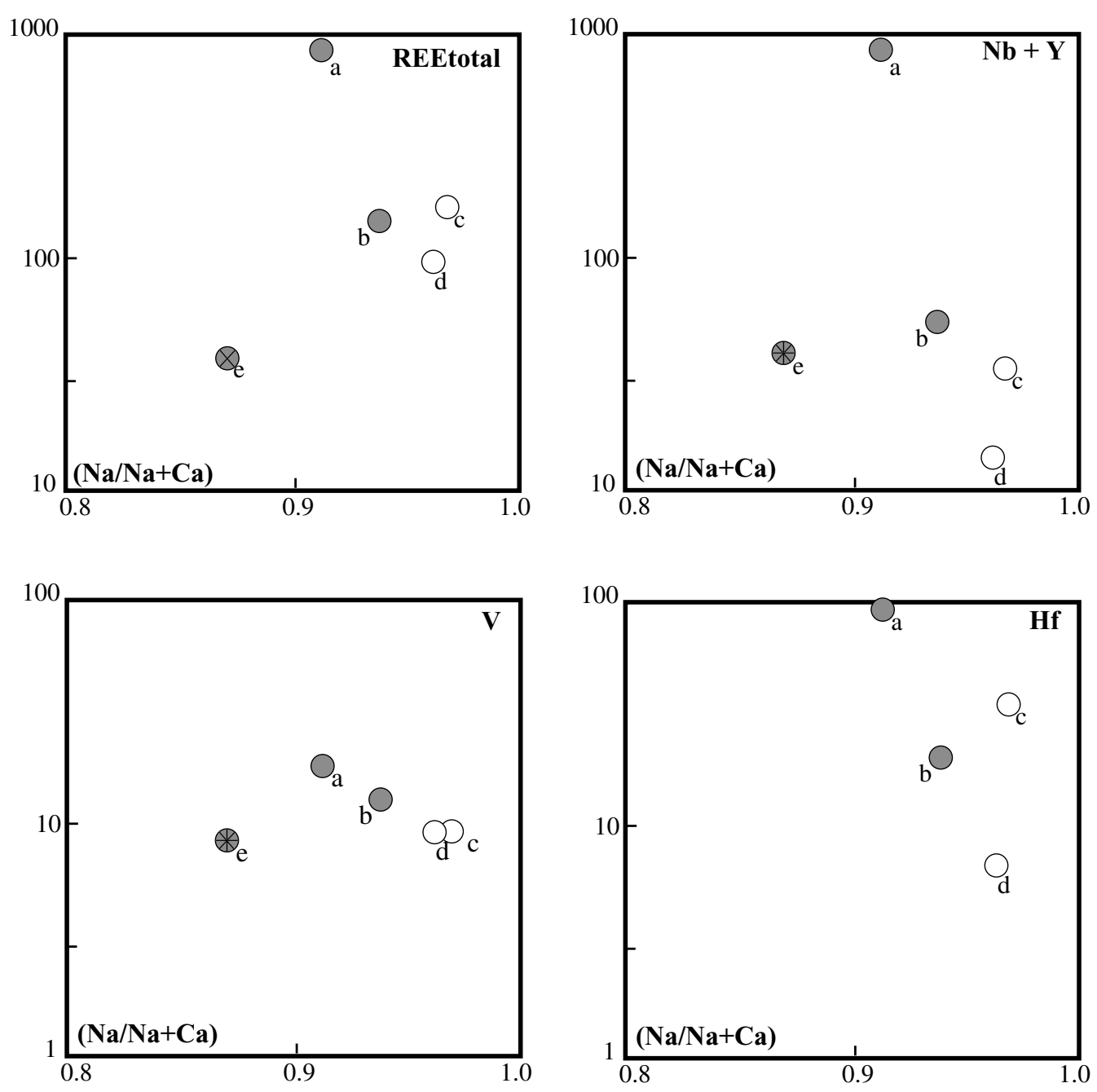

Fig. 19. Trace elements vs. $\mathrm{Na} /(\mathrm{Na}+\mathrm{Ca})$ diagrams in Ti-rich aegirine of the strongly peralkaline granites. The identified analyses are the same as Fig. 17B and Tables 2-6.

or astrophyllite). Astrophyllite has been described by Stephenson and Upton (1982), showing that this mineral is an accessory phase commonly associated with the paragenesis Na-amphibole, Na-pyroxene, biotite and zircon. According to MacDonald and Saunders (1973) astrophyllite is a late magmatic mineral probably produced by low temperature reaction involving ilmenite and alkali-rich residual fluid (Stephenson and Upton, 1982). In the Serra do Meio suite, this paragenesis is produced at the same subsolidus temperature suggested for the metamorphic reaction riebeckite + quartz + fluid $\rightarrow$ aegirine + magnetite + quartz + fluid.

Regarding Fig. 20B, riebeckite-arfvedsonite crystals are stable under more reduced conditions than pure riebeckite, and a temperature around $700^{\circ} \mathrm{C}$. Similar conditions were assumed by Fabriès (1978) and Bonin (1982), who have shown that riebeckite-arfvedsonite grains crystallise under the most reduced conditions among the Fe-rich alkali amphiboles.

\section{Conclusions}

The Paleoproterozoic Serra do Meio alkaline, oversaturated suite was emplaced in the Riacho do Pontal Fold Belt, northeast Brazil, and consists of metaluminous, slightly peralkaline and strongly peralkaline granites. Metamorphism of the metaluminous and strongly peralkaline granites is close to greenschist and low-amphibolite limit. The metamorphic paragenesis formed during the Neoproterozoic event were superimposed on the original magmatic assemblages.

Metaluminous granites are characterised by annite whereas the slightly peralkaline granites contain aegirineaugite and aegirine as their chief mafic components. In the strongly peralkaline granites, the crystallisation sequence: riebeckite-winchite $\rightarrow$ Ti-aegirine-augite $\rightarrow$ Ti-aegirine was observed. The Ti-rich pyroxene shows strong enrichment in incompatible elements, notably $\mathrm{Nb}, \mathrm{Y}$, and REE, with patterns comparable to those described by Larsen 

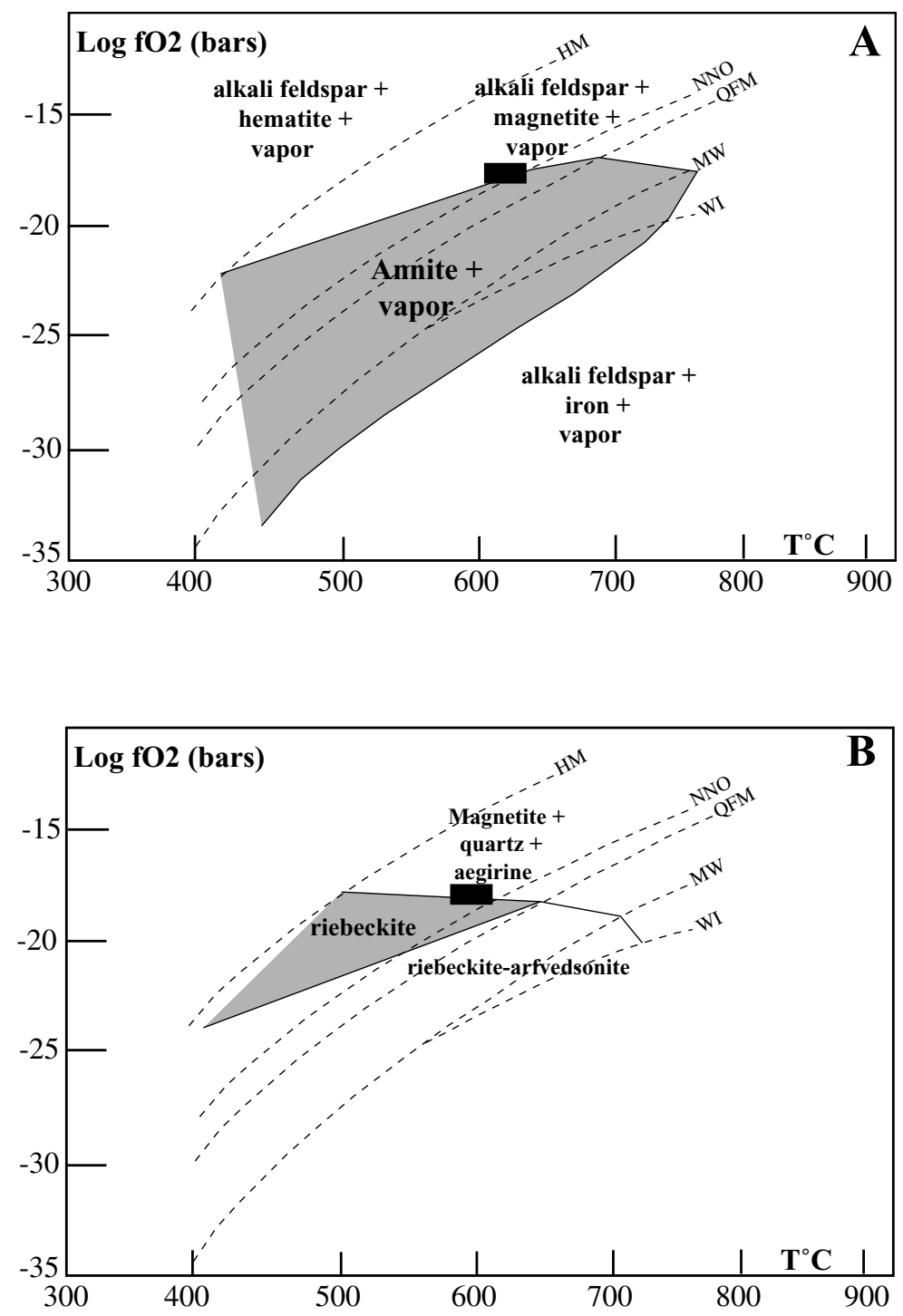

Fig. 20. $\mathrm{Log} \mathrm{fO}_{2}$ vs. $T$ diagrams at $P=2 \mathrm{kbar}$, showing the probable upper metamorphic limit (filled rectangles) for (A) metaluminous granites and (B) strongly peralkaline granites. Mineral stability fields: Eugster and Wones (1962) in figure A and Ernst (1962) in figure B.

(1979) and Shearer and Larsen (1994). In the strongly peralkaline granites, $\mathrm{Nb}$ and $\mathrm{Y}$ have been removed by alkali amphiboles and pyroxenes. In the slightly peralkaline facies, these elements were partitioned between aegirine and exotic REE- $\mathrm{Nb}-\mathrm{Y}-\mathrm{F}$ exotic minerals.

During the Brasiliano event, the following metamorphic reactions were identified: (i) microcline $+\mathrm{Fe}$-rich fluid + quartz $\rightarrow$ annite + magnetite + quartz and riebeckite + quartz + Fe-rich $\quad$ fluid $\rightarrow$ aegirine + magnetite + quartz, both suggesting the upper limit of metamorphism and (ii) Tipyroxene $\rightarrow$ riebeckite + titanite + (astrophyllite or aenigmatite). Metamorphism generated magnetite, low-Fe annite, and pure riebeckite, while causing pyroxene to lose $\mathrm{Ti}$ and incompatible elements such as REE and $\mathrm{Nb}$. Metamorphic conditions were close to the NNO buffer and temperatures around $600^{\circ} \mathrm{C}$ (Fig. $20 \mathrm{~A}$ and B). Fe-rich fluids has played a significant role in producing the metamorphic paragenesis.

\section{Acknowledgements}

J.P.C. thanks the Fundação Coordenação de Aperfeiçoamento de Pessoal de Nível Superior (CAPES-n. 1772/95-14), CNPq for financial support, Centro de Estudos em Petrologia e Geoquímica-UFRGS, Programa de Pesquisa e Pós-Graduação em Geofísica, PPPGUFBA, Laboratoire de Petrographie et Volcanologie, Centre d'Orsay, Paris XI, the Prof. Dr. Léo Afraneo Hartmann for the paper revision and his wife, for the patience.

\section{References}

Barbosa, J.S.F., Dominguez, J.M.L., 1996. Mapa geológico do Estado da Bahia. Texto Explicativo. Secretaria da Indústria, Comércio e Mineração do Estado da Bahia. PPPG-UFBA (Ed.). Salvador. 
Bonin, B., 1980. Le complexes acides alcalins anorogéniques continentaux: l'exemple de la Corse. Doct. Etat ès-Sci. Thèses, Université Pierre et Marie Curie, Paris.

Bonin, B, 1982. Les granites des complexes annulaires. Manuels et Méthodes no. 4, B.R.G.M. (Ed.), Orléans.

Bonin, B., 1988. Peralkaline granites in Corsica: some petrological and geochemical constraints. Rendicontti Della Societa Italiana de Mineralogia i Petrologia 43, 281-306.

Bonin, B., Giret, A., 1985. Clinopyroxene compositional trends in oversaturated and undersaturated alkaline ring complexes. Journal of African Earth Sciences 3, 175-183.

Bowden, P., 1982. Magmatic evolution and mineralization in the Nigerian Younger Granite province. In: Evans, M.A. (Ed.). Metallization Associated with Acid Magmatism. Wiley, New York, pp. 51-61.

Bowden, P., Kinnaird, J.A., 1984. The petrology and geochemistry of alkaline granites from Nigeria. Physics and Earth Planetary Interiors 35, 199-211.

Boyd, F.R., 1959. Hydrothermal investigations of amphiboles. Journal of Petrology 14 (3), 349-380.

Brito Neves, B.B., 1975. Regionalização geotectônica do Pré-Cambriano nordestino. Tese de Doutorado, Instituto de Geociências, Universidade de São Paulo.

Cameron, M., Sueno, S., Prewitt, C.T., Papike, J.J., 1973. High-temperature crystal chemistry of acmite, diopside, hedenbergite, jadeite, spodumene, and ureyite. American Mineralogy 58, 594-619.

Clark, J.R., Appleman, D.E., Papike, J.J., 1969. Crystal-chemical characterization of clinopyroxene based on eight new structure refinements. Mineralogical Society of American Special Paper 2, 31-50.

Conceição, H., 1990. Pétrologie du massif syénitique d'Itiúba: contribution à l'étude minéralogique des roches alcalines dans l'Etat de Bahia (Brésil). Tese de Doutorado, Université Paris-Sud, Paris.

Conceição, R.V., 1994. Petrologia dos sienitos potássicos do maciço de Santanapólis e alguns aspectos do seu embasamento granulítico. Dissertação de Mestrado, PPPG-UFBA, Salvador.

Couto, L.F., 1989. Estudo petrológico do complexo máfico-ultramáfico de Campo Alegre de Lourdes e dos óxidos de Fe, Ti, (V) associados. Dissertação de Mestrado, Universidade de Brasília-UNB, Brasília.

Czamanske, G.K., Wones, D.R., 1973. Oxidation during magmatic differentiation, Finnmarka Complex, Oslo area, Norway: Part 2, the mafic silicates. Journal of Petrology 14 (3), 349-380.

Dalton de Souza, J.A., Fernandes, F.J., Guimarães, J.T., Lopes, J.N., 1979. Projeto Colomi: Geologia da região do Médio São Francisco. Relatório final. Salvador, CPRM. Convênio DNPM/CPRM.

Dooley, D.F., Patiño Douce, A.E., 1996. Fluid-absent melting of F-rich phlogopite + rutile + quartz. American Mineralogist 81, 202-212.

Dorais, M.J., Floss, C., 1992. An ion and Electron Microprobe Study of the Mineralogy of Enclaves and Host Syenites of the Red Hill Complex, New Hampshire, USA. Journal of Petrology 33 (5), 1193-1218.

Ernst, W.G., 1962. Synthesis, stability relations, and occurrence of riebeckite and riebeckite-arfvedsonite solid solutions. Journal of Geology 70, 689-736.

Eugster, H.P., Wones, D.R., 1962. Stability relations of a ferrugineous biotite, annite. Journal of Petrology 3, 82-125.

Evensen, N.M., Hamilton, P.J., O'Nions, R.K., 1978. Rare earth abundances in chondritic meteorites. Geochimica et Cosmochimica Acta 42, 1199-1212.

Fabriès, J., 1978. Les types paragenénétiques des amphiboles sodiques dans les roches magmatiques. Bulletim Mineralogique 101, 155-165.

Ferguson, A.K., 1977. The natural occurrence of aegirine-neptunite solid solution. Contributions to Mineralogy and Petrology 60, 247-253.

Ferguson, A.K., 1978. The occurrence of ramsayite, titan-lavenite and a fluorine-rich eucolite in a nepheline-syenite inclusion from Tenerife, Canary Islands. Contributions to Mineralogy and Petrology 66, 15-20.

Giret, A., Bonin, B., Leger, J.M., 1980. Amphibole compositional trends in over saturated and undersaturated alkaline plutonic ring complexes. The Canadian Mineralogist 18, 481-485.

Halliday, A.N., Davidson, J.P., Hildreth, W., Holden, P., 1991. Modelling the petrogenesis of high $\mathrm{Rb} / \mathrm{Sr}$ silicic magmas. Chemical Geology 92, $107-114$

Harris, N.B.W., 1980. The role of fluorine and chlorine in the petrogenesis of a peralkaline complex from Saudi Arabia. Chemical Geology 31, 303-310.

Jacobson, R.R.E., Mac Leod, W.N., Black, R., 1958. Ring-complexes in the Younger Granite Province of northern Nigeria. Geological Society of London Memoir 1, 1-72.

Jardim de Sá, E.F., 1994. A faixa Seridó (Província Borborema, NE do Brasil) e o seu significado geodinâmico na cadeia Brasiliana/PanAfricana, Tese de Doutorado, UnB, Brasília.

Larsen, L.M., 1976. Clinopyroxene and coexisting mafic minerals from the alkaline Ilímaussaq intrusion, South Greenland. Journal of Petrology $17,258-290$.

Larsen, L.M., 1977. Aenigmatites from the Ilímaussaq intrusion, South Greenland. Lithos 10, 257-270.

Larsen, L.M., 1979. Distribution of REE and other trace elements between phenocrysts and peralkaline undersaturated magmas, exemplified by rocks from the Gardar igneous province, South Greenland. Lithos 12, 303-315.

Leake, B.E., 1978. Nomenclature of amphibole. Mineralogical Magazine 42, 533-563.

Leake, B.E., Wooley, A.R., Arps, C.E.S., Birch, W.D., Gilbert, M.C., Grice, J.D., Hawthorne, F.C., Kato, A., Kisch, H.J., Krivovichev, V.G., Linthout, K., Laird, J., Mandarino, J.A., Maresch, W.V., Nickel, E.H., Rock, N.M.S., Schumacher, J.C., Smith, D.C., Stephenson, N.C.N., Ungaretti, L., Whittaker, E.J.W., Youzhi, G., 1997. Nomenclature of amphiboles: report of the subcommittee on amphiboles of the international mineralogical association. Commission on new minerals and mineral names. American Mineralogist 82, 1019-1037.

Leite, C.M., 1987. Projeto Remanso II. Relatório Final, CBPM-SME, Salvador.

Leite, C.M., 1997. Programa de Levantamentos Geológicos Básicos do Brasil, Folha-SC.23-X-D-IV (Campo Alegre de Lourdes), escala 1:100000. CBPM/CPRM/SICM-Salvador.

Leite, C.M., Conceição, H., Cruz, M.J., 1991. Plutonismo hiperalcalino supersaturado da Província de Campo Alegre de Lourdes: Evolução mineraloquímica e suas implicações. III Congresso Brasileiro de Geoquímica, São Paulo. Anais 2, 717-721.

Leite, C.M.M., Santos, R.A., Conceição, H., 1993. A província toleíticaalcalina de Campo Alegre de Lourdes: geologia e evolução tectônica. II Simpósio sobre o Cráton do São Francisco. SBG/SGM, Salvador. Anais $1,56-59$.

1989. A Classification of Igneous Rocks and Glossary of Terms. In: Le Maitre, R.W., Bateman, P., Dubek, A., Keller, J., Lameyre, J., Le Bas, M.J., Sabine, P.A., Schimid, R., Sorensen, H., Streckeisen, A., Wooley, A.R., Zanettin, B. (Eds.). Recommendations of the International Union of Geological Sciences Subcommission on the Systematics of Igneous Rocks. Blackwell, Oxford.

MacDonald, R., Saunders, M.J., 1973. Chemical variation in minerals of the astrophyllite group. Mineralogical Magazine 39, 97-111.

Martin, R.F., Piwinskii, A.J., 1972. Magmatism and tectonic settings. Journal of Geophysical Research 77, 4966-4975.

Middlemost, E.A.K., 1994. Naming materials in the magma/igneous rock system. Earth Science Review 37, 215-224.

Mitchell, R.H., 1990. A review of the compositional variation of amphiboles in alkaline plutonic complexes. Lithos 26, 135-156.

Miyashiro, A., 1957. The chemistry, optics, and genesis of the alkaliamphiboles. Journal of Faculty of Science Sector II 11 (1), 57-83 (University of Tokyo).

Morimoto, C.N., 1988. Nomenclature of pyroxenes. Mineralogical Magazine 52, 535-550.

Murthy, M.V.N., Venkatenaman, P.K., 1964. Petrogenetic significance of certain platform peralkaline granites of the world. The Upper Mantle Symposium. New Delhi, pp. 127-149.

Nachit, Razafimahefa, N., Stussi, J., Carron, J., 1985. Composition chimique des biotites et typologie magmatique des granitoids. Compte 
Rendus de l'Academie des Sciences de Paris t. 301, serie II, vol. 11, pp. 813-818.

Nardi, L.V.S., 1991. Caracterização petrográfica e geoquímica dos granitos metaluminosos da associação alcalina: revisão. Pesquisas 18 (1), 44-57.

Nardi, L.V.S., Bonin, B., 1991. Post-orogenic and non-orogenic alkaline granite associations: the Saibro Intrusive Suite, Southern Brazil - a case study. Chemical Geology 92, 197-212.

Neumann, E.R., 1976. Compositional relations among pyroxenes, amphiboles and other mafic phases in the Oslo Region plutonic rocks. Lithos 9, 85-109.

Nielsen, T.E.D., 1979. The occurrence and formation of Ti-aegirines in peralkaline syenites: an example from the Tertiary Ultramafic Alkaline Gardiner Complex, East Greenland. Contributions to Mineralogy and Petrology 69, 235-244.

Nockolds, S.R., 1947. The relation between chemical compositions and paragenesis in the biotite micas of igneous rocks. American Journal of Science 245, 401-420.

Paim, M.M., 1998. Petrologia da intrusão sienítica potássica de Cara Suja (Sudoeste da Bahia). Dissertation Mestrado, Pós-Graduação em Geoquímica e Meio Ambiente./UFBA. Salvador.

Patiño Douce, A.E., Beard, J.S., 1996. Effects of $\mathrm{P}, \mathrm{f}\left(\mathrm{O}_{2}\right)$ and $\mathrm{Mg} / \mathrm{Fe}$ ratio on dehydration melting of model metagreywackes. Journal of Petrology 37 (5), 999-1024.

Pearce, J.A., Harris, N.B.W., Tindle, A.G., 1984. Trace element discrimination diagrams for the tectonic interpretation of granitic rocks. Journal of Petrology 25, 956-983.

Plá Cid, J., 1994. Granitogênese alcalina de Campo Alegre de Lourdes (Norte da Bahia): Petrografia, Mineraloquímica e Geoquímica. Dissertação de Mestrado, PPPG-UFBA, Salvador.

Plá Cid, J., Conceição, H., Nardi, L.V.S., 1995. As micas tri-octaédricas da suíte granítica de Campo Alegre de Lourdes (N da Bahia). V Congresso Brasileiro de Geoquímica e III Congresso Geoquímica dos Países de Língua Portuguesa, Niterói, CD-Rom.

Plá Cid, J., Nardi, L.V.S., Conceição, H., Bonin, B., 1997. O magmatismo alcalino da faixa de dobramentos Riacho do Pontal e da borda noroeste do Cráton do São Francisco, norte do Estado da Bahia, Brasil: uma síntese. X Semana de Geoquímica e IV Congresso de Geoquímica dos Países de Língua Portuguesa, Braga — Portugal. Actas, 127-130.

Plá Cid, J., Nardi, L.V.S., Conceição, H., Bonin, B., 1999. The magmatic evolution of ultrapotassic syenite-granite suites, northeastern Brazil: a major and trace element approach. International Geology Review 4 (11), 1005-1028.

Plá Cid, J., Bitencourt, M.F., Nardi, L.V.S., Conceição, H., Bonin, B., Lafon, J.M., 2000. Paleoproterozoic anorogenic and late-orogenic alkaline granitic magmatism from northeast Brazil. Precambrian Research $104(1 / 2), 47-75$.

Rieder, M., Cavazzini, G., D’Yakonov, Y.S., Frank-Kamenetskii, V.A., Gottardi, G., Guggenheim, S., Koval, P.V., Muller, G., Neiva,
A.M.R., Radoslovich, E.W., Robert, J.-L., Sassi, F.P., Takeda, H., Weiss, Z., Wones, D.R., 1998. Nomenclature of Micas. The Canadian Mineralogist 36 (3), 905.

Rios, D.C., 1997. Petrologia do magmatismo potássico-ultrapotássico e lamprofírico de Morro do Afonso, Bahia. Dissertation Mestrado, Curso de Pós-Graduação em Geologia-UFBA, Salvador.

Rogers, J.J.W., Greenberg, J.K., 1990. Late-orogenic, post-orogenic and anorogenic granites: distinction by major-element and trace-element chemistry and possible origins. Journal of Geology 98 (3), 291-309.

Rosa, M.L.S., 1994. Magmatismo shoshonítico e ultrapotássico no sul do cinturão móvel Salvador-Curaçá, maciço de São Félix: geologia, mineralogia e geoquímica. Dissertação de mestrado, CPGG-UFBA, Salvador.

Rutherford, M.J., 1969. An experimental determination of iron biotitealkali feldspar equilibria. Journal of Petrology 10, 381-408.

Shearer, C.K., Larsen, L.M., 1994. Sector-zoned aegirine from the Ilímaussaq alkaline intrusion, South Greenland: implications for trace-element behavior in pyroxene. American Mineralogist 79, 340-352.

Shearer, C.K., Papike, J.J., Simon, S.B., Galbreath, K.G., Shimizu, N., 1989. An ion microprobe study of the intra-crystalline behavior of REE and selected trace elements in pyroxene from mare basalts with different cooling and crystallization histories. Geochimica et Cosmochimica Acta 53, 1041-1054.

Silva, A.B., Liberal, G.S., Grossi Sad, J.M., Issa Filho, A., Rodrigues, C.S., Riffel, D.F., 1988. Geologia e Petrologia do Complexo Angico dos Dias (Bahia, Brasil), uma associação carbonatítica précambriana. Geochimica Brasiliensis 2 (1), 81-108.

Sorensen, H., 1974. The Alkaline Rocks. Wiley, London.

Stephenson, D., Upton, B.G.J., 1982. Ferromagnesian silicates in a differentiated alkaline complex: Kûngnât Fjeld, South Greenland. Mineralogical Magazine 46, 283-300.

Streckeisen, A., 1976. To each plutonic rocks its proper name. Earth Science Review 12, 1-33.

Upton, B.G.J., 1974. The alkaline province of south-west Greenland. In: Sorensen, H. (Ed.). The Alkaline Rocks. Wiley, New York, pp. 221-238.

Vannucci, R., Tribuzio, R., Piccardo, G.B., Ottolini, L., Bottazzi, P., 1991. SIMS analyses of REE in pyroxenes and amphiboles from the Proterozoic Ikasaulak intrusive complex (SE Greenland): implications for LREE enrichment processes during post-orogenic plutonism. Chemical Geology 92, 115-133.

Vorma, A., 1976. On the petrogeochemistry of Rapakivi granites with special reference to the Laitila massif, southwestern Finland. Bulletin of Geological Survey of Finland 285, 98.

Watson, E.B., 1979. Zircon saturation in felsic liquids: experimental results and applications to trace element geochemistry. Contributions to Mineralogy and Petrology 70, 407-419.

Wernick, 1981. The Archean of Brazil. Earth-Science Reviews 17, 31-48.

Whalen, J.B., Currie, K.L., Chappell, B.W., 1987. A-type granites: geochemical characteristics, discrimination and petrogenesis. Contributions to Mineralogy and Petrology 95, 407-419. 